\title{
Musical intervals under 12-note equal temperament: a geometrical interpretation
}

\author{
R. Caimmi*†, M A. Franzon ${ }^{\ddagger}$, S. Tognon
}

February 2, 2017

\begin{abstract}
Musical intervals in multiple of semitones under 12-note equal temperament, or more specifically pitch-class subsets of assigned cardinality, $n, 1 \leq n \leq 12$, (n-chords) are conceived as positive integer points, $\mathrm{P}_{\mathrm{n}} \equiv\left(\ell_{1}, \ell_{2}, \ldots, \ell_{n}\right), \ell_{1}+\ell_{2}+\ldots+\ell_{n}=12$, within an Euclidean $n$ space, $\Re^{n}$. The number of distinct $n$-chords, $N_{\mathrm{C}}(n)$, is inferred from combinatorics with the extension to $n=0$, involving an Euclidean 0 -space, $\Re^{0}$. The number of repeating $n$-chords, $\Delta N(n)$, or points which are turned into themselves during a circular permutation, $T_{n}$, of their coordinates, is inferred from algebraic considerations. Finally, the total number of $n$-chords, $N_{\mathrm{M}}(n)=N_{\mathrm{C}}(n)+\Delta N(n)$, and the number of $T_{n}$ set classes, $\nu_{\mathrm{M}}(n)=N_{\mathrm{M}}(n) / n$, are determined. Palindrome and pseudo palindrome $n$-chords are defined and included among repeating $n$-chords, with regard to an equivalence relation, $T_{n} / T_{n} I$, where reflection is added to circular permutation. To this respect, the number of $T_{n}$ set classes is inferred concerning palindrome
\end{abstract}

\footnotetext{
*Physics and Astronomy Department, Padua University, Vicolo Osservatorio 3/2, 35122 Padova, Italy email: roberto.caimmi@unipd.it fax: 39-049-8278212

${ }^{\dagger}$ Affiliated up to September 30th 2014. Current status: Studioso Senior. Current position: in retirement due to age limits.

${ }^{\ddagger}$ Associazione Culturale S. Nicolò, Piazza Pio X 27, 36043 Camisano Vicentino (VI), Italy email: albertfranzmuu@gmail.com

$\S$ Via Fogazzaro 32, 36043 Camisano Vicentino (VI), Italy

ฯFBD House, Bluebell, Dublin 12, Ireland email: stetogno@gamil.com fax:+353
} (0) 14783574 
and pseudo palindrome $n$-chords, $\nu_{\mathrm{P}}(n)$, and the remaining $n$-chords, $\nu_{\mathrm{N}}(n)=\nu_{\mathrm{M}}(n)-\nu_{\mathrm{P}}(n)$, yielding a number of $T_{n} / T_{n} I$ set classes, $\nu_{\mathrm{Q}}(n)=\nu_{\mathrm{N}}(n) / 2+\nu_{\mathrm{P}}(n)$. The above results are reproduced within the framework of a geometrical interpretation, where positive integer points related to $n$-chords of cardinality, $n$, belong to a regular inclined $n$-hedron, $\Psi_{12}^{n}$, the vertexes lying on the coordinate axes of a Cartesian orthogonal reference frame, $\left(\mathrm{O} x_{1} x_{2} \ldots x_{n}\right)$, at a distance, $x_{i}=12,1 \leq i \leq n$, from the origin. Considering $\Psi_{12}^{n}$ as special cases of lattice polytopes, the number of related nonnegative integer points is also determined for completeness. A comparison is performed with the results inferred from group theory. The symmetry of the number of $n$-chords, $T_{n}$ set classes, $T_{n} / T_{n} I$ set classes, with regard to cardinality, is interpreted as intrinsic to $n$-hedrons and, for this reason, expressed via the binomial formula. More generally, the symmetry of the results inferred from the group theory could be conceived as intrinsic to lattice polytopes in $\Re^{n}$.

Keywords: pitch-classes; $n$-chords; $T_{n}$ set classes; $T_{n} / T_{n} I$ set classes; Euclidean $n$-spaces; $n$-hedrons

\section{Introduction}

The question of how many musical intervals in multiples of semitones there are under 12-note equal temperament (more specifically, how many pitchclass subsets there are of a given cardinality, or how many there are with respect to one fixed pitch-class and further organized under various equivalence relations) is one that has been answered early and often in the music theory and mathematical literature e.g., [1] [2]. The problem has been worked out independently, in particular the partition into equivalence classes under transposition (or circular permutation) e.g., [3] 4] 5]. To this respect, it is worth emphasyzing combinatorial problems are not essentially musical: the same procedure can be applied e.g., for the isomer enumeration in chemistry, for spin analysis in physics, and in general for the investigation of isomorphism classes of objects e.g., [6] 7]. The most elegant way for solving such problems is the Polya-Burnside method, which was applied to music theory more than thirty years ago [8].

Both pitch-class subsets and $T_{n}$ set classes of each cardinality from 0 through 12 are familiar to music theorists but the set-class counts, in absence of a deep knowledge of the group theory, are performed by use of tables enumerating all the set classes e.g., [9] [3] [4][10] [11]. An intermediate use between the two extremes mentioned above relates to standard techniques in classical combinatorial theory and offers some simple applications to music theory, in- 
cluding the enumeration of pitch-class subsets e.g., [12] 13] 15]. In addition, following this line of thought foreshadows certain aspects of the more difficult work involved in group theory, and therefore may form a pedagogically benefical bridge to the advanced material e.g., [14] Chap. 9 [15] 17].

To this respect, the present paper is restricted to the simplest case of $T_{n}$ and $T_{n} / T_{n} I$ set classes, with regard to pitch-class subsets (internal patterns or internal structures) of cardinality, $n$, where the sum of musical intervals in multiples of semitones equals 12 , or $n$-chords. Of course, related results are already known in the literature e.g., 8] [14] Chap. 9 [15] 16] 17] [19], but the exposition here is expected to be more readily accessible to music theory community and, last but not least, to interest in group theory by itself. The current approach is essentially algebraic and geometric: in short, the paper presents "an algorithmic theory," one of many possible. The main steps of the method may be summarized as follows.

First, $n$-chords, $\left\{\ell_{1}, \ell_{2}, \ldots \ell_{n}\right\}$, are conceived as positive integer points of coordinates, $\mathrm{P}_{\mathrm{n}} \equiv\left(\ell_{1}, \ell_{2}, \ldots \ell_{n}\right)$, with respect to a Cartesian orthogonal reference frame, $\left(\mathrm{O} x_{1} x_{2} \ldots x_{n}\right)$, in an Euclidean $n$-space, $\Re^{n}$. In this view, $n$-chords may be thought of as made of coordinates. Then $T_{n}$ set classes of each cardinality are partitioned into two main categories, namely set classes where $n$-chords exhibit distinct e.g., $(1,2,3,6)$ and repeating e.g., $(2,4,2,4)$ coordinates, respectively.

Second, $n$-chords belonging to the above mentioned categories are enumerated separately and the amount of related $T_{n}$ set classes is determined.

Third, the number of $T_{n} / T_{n} I$ set classes of each cardinality is also determined following a similar procedure.

Fourth, further attention is devoted to the geometrical interpretation in itself.

The method could, in principle, be extended to musical intervals in multiples of semitones under $L$-note (instead of 12-note) equal temperament.

The text is organized as follows. The first, second and third step outlined above are developed in different subsections of Section 2. The fourth step is considered in Section 3. The discussion is presented in Section 4. The conclusion is shown in Section 5. As guidance examples, the method is applied to classical birthday-cake and necklace problem in Appendix $\mathrm{A}$ and B. respectively. General properties of $n$-hedrons are outlined in Appendix C. 


\section{Enumeration of $n$-chords, $T_{n}$ and $T_{n} / T_{n} I$ set classes}

A pitch-class subset is defined to be a subset of the set of twelve pitch-classes e.g., [14] Chap. 9 §9.14. In musical terms, natural numbers within the range, $1 \leq n \leq 12$, could be thought of as representing musical intervals in multiples of semitones, in the twelve tone equal tempered octave. Octave equivalence in the musical scale implies two notes belong to the same pitch-class if they differ by a whole number of octaves. Then addition has an obvious interpretation as addition of musical intervals. To this respect, an origin must be chosen via one fixed pitch-class. For further details, an interested reader is addressed to specific investigations e.g., [17] or textbooks e.g., [14] Chap. 9.

Accordingly, pitch-class subsets of cardinality, $n$, are denoted as $n$-tuples, $\left\{\ell_{1}, \ell_{2}, \ldots, \ell_{n}\right\}$, where $\ell_{1}, \ell_{2}, \ldots, \ell_{n}$, are natural numbers. Let $n$-chords be defined as pitch-class subsets where the boundary condition:

$$
\ell_{1}+\ell_{2}+\ldots+\ell_{n}=12 ; \quad 1 \leq n \leq 12 ;
$$

is satisfied e.g., [17].

$T_{n}$ set classes are obtained by transposition (or circular permutation) as $\left\{\ell_{1}, \ell_{2}, \ldots, \ell_{n}\right\},\left\{\ell_{2}, \ell_{3}, \ldots, \ell_{1}\right\}, \ldots,\left\{\ell_{n}, \ell_{1}, \ldots, \ell_{n-1}\right\} . T_{n} / T_{n} I$ set classes are obtained by reflection (or order inversion) followed by a transposition. More specifically, the application of the pitch-class operator, $T_{k}, k \leq n$, on the $n$ tuple, $\left\{\ell_{1}, \ell_{2}, \ldots, \ell_{n}\right\}$, yields $\left\{\ell_{k+1}, \ldots, \ell_{n}, \ell_{1}, \ldots, \ell_{k}\right\}$, and the application of the pitch-class operator, $T_{k} I$, on the same $n$-tuple, yields a reflection, $\left\{\ell_{n}, \ell_{n-1}, \ldots, \ell_{1}\right\}$, followed by a transposition, $\left\{\ell_{k}, \ldots, \ell_{1}, \ell_{n}, \ldots, \ell_{k+1}\right\}$.

Let the prime form of a set class be defined as a special $n$-chord within that class, for which (i) the last element of the $n$-tuple has the larger value and, in case of multiplicity, (ii) the first element of the $n$-tuple has the lower value [20]. For instance, the prime form of the $T_{n} / T_{n} I$ set class,

$$
\{1,2,9\},\{2,9,1\},\{9,1,2\},\{1,9,2\},\{9,2,1\},\{2,1,9\}
$$

is $\{1,2,9\}$. Accordingly, $T_{n}$ and $T_{n} / T_{n} I$ set classes can be represented by their prime forms, as cyclic "adjacent internal array" $\left(\mathrm{CINT}_{1}\right)$ [20].

Let $n$-chords be defined as "distinct" and "repeating" according if related $n$-tuples are different or coinciding, respectively. Let $T_{n}$ set classes be defined as "distinct" and "repeating" according if related $n$-chords are distinct or repeating, respectively. For instance, the $T_{n}$ set class,

$$
\{1,2,3,6\},\{2,3,6,1\},\{3,6,1,2\},\{6,1,2,3\} ;
$$


is made of four distinct 4-chords, while the $T_{n}$ set class,

$$
\{1,5,1,5\},\{5,1,5,1\},\{1,5,1,5\},\{5,1,5,1\} ;
$$

is made of two distinct and two repeating 4-chords. It is worth mentioning repeating musical intervals in multiples of semitones have been studied and used since about 70 years ago [12].

A method shall be exploited in the following subsections, where distinct and repeating $n$-chords shall be counted separately to yield the number of $T_{n}$ and $T_{n} / T_{n} I$ set classes.

\subsection{Enumeration of distinct $n$-chords}

Aiming to a geometrical interpretation, $n$-tuples representing $n$-chords shall be considered as coordinates of points, $\mathrm{P}_{\mathrm{n}} \equiv\left(\ell_{1}, \ell_{2}, \ldots, \ell_{n}\right)$, with respect to a Cartesian orthogonal reference frame, $\left(\mathrm{O} x_{1} x_{2} \ldots x_{n}\right)$, in an Euclidean $n$ dimension hyperspace, or $n$-space, $\Re^{n}$. More specifically, $\mathrm{P}_{\mathrm{n}}$ lies within the positive $2^{n}$-ant (2-ant is versant, 4 -ant is quadrant, 8 -ant is octant, and so on), and the coordinates are natural numbers linked via Eq. (1).

With no loss of generality, the dependent coordinate may be chosen to be $\ell_{n}$, as:

$$
\ell_{n}=12-\ell_{1}-\ell_{2}-\ldots-\ell_{n-1} ; \quad 1 \leq n \leq 12 ;
$$

and the projection of $\mathrm{P}_{\mathrm{n}}$ onto the principal $(n-1)$-dimension hyperplane, or $(n-1)$-plane, $\left(\mathrm{O} x_{1} x_{2} \ldots x_{n-1}\right)$, is $\mathrm{P}_{\mathrm{n}-1} \equiv\left(\ell_{1}, \ell_{2}, \ldots, \ell_{n-1}\right)$. The knowledge of $P_{n-1}$ implies the knowledge of $P_{n}$ via Eq. (2).

Given a generic projected point, $\mathrm{P}_{\mathrm{n}-1} \equiv\left(\ell_{1}, \ell_{2}, \ldots, \ell_{n-2}, \ell_{n-1}\right)$, let the conjugate point be defined as $\mathrm{Q}_{\mathrm{n}-1} \equiv\left(12-\ell_{1}, S_{2, n-1}, \ldots, S_{n-2, n-1}, \ell_{n-1}\right)$, where, in general, $S_{i, n-k}$ are expressed as:

$$
\begin{aligned}
& S_{i, n-k}=\ell_{i}+\ell_{i+1}+\ldots+\ell_{n-k-1}+\ell_{n-k} ; \\
& 0<\ell_{n-1}<S_{n-2, n-1}<\ldots<S_{3, n-1}<S_{2, n-1}<12-\ell_{1} \\
& 0<\ell_{i}<12 ; \quad 1 \leq i \leq n-1 ;
\end{aligned}
$$

and Eq. (3b) follows from (11), (3c).

According to Eq. (3), the projected points, $P_{n-1}, Q_{n-1}$, are in a $1: 1$ correspondence, $\mathrm{P}_{\mathrm{n}-1} \leftrightarrow \mathrm{Q}_{\mathrm{n}-1}$, or:

$$
\left(\ell_{1}, \ell_{2}, \ldots, \ell_{n-2}, \ell_{n-1}\right) \leftrightarrow\left(12-\ell_{1}, S_{2, n-1}, \ldots, S_{n-2, n-1}, \ell_{n-1}\right) ;
$$

where the coordinates on the right-hand side of Eq. (4) are clearly distinct, monotonically decreasing, and belonging to the subset of natural numbers, $\{1,2, \ldots, 11\}$, via Eq. (3). The special case, $n=3$, is shown in Fig.1, 
With regard to the projected point, $\mathrm{Q}_{\mathrm{n}-1}$, there are $11-n+n=11-0$ different ways of choosing the first coordinate, $11-n+(n-1)=11-1$ different ways of choosing the second coordinate with the preceeding fixed, $\ldots, 11-n+[n-(n-2)]=11-n+2$ different ways of choosing the $(n-1)$ th coordinate with the preceeding fixed, for a total, $N_{\mathrm{C}}^{\prime}=11 \cdot 10 \cdot \ldots \cdot(11-n+$ $2)=11 ! /[11-(n-1)]$ !, including points whose coordinates are linked by permutations i.e. with place exchanged one with respect to the other.

For $(n-1)$ fixed distinct coordinates, there are $(n-1)$ different ways of choosing the first coordinate, $(n-2)$ different ways of choosing the second coordinate with the preceeding fixed, ..., $[n-(n-2)]=2$ different ways of choosing the $(n-2)$ th coordinate with the preceeding fixed, $[n-(n-1)]=1$ univocal way of choosing the $(n-1)$ th coordinate with the preceeding fixed, for a total, $N^{\prime}=(n-1) \cdot(n-2) \cdot \ldots \cdot 2 \cdot 1=(n-1)$ !.

In conclusion, the total number of projected points, $Q_{n-1}$, having coordinates (i) belonging to the subset of natural numbers, $\{1,2, \ldots, 11\}$; (ii) distinct the one with respect to the other; (iii) univocally ordered i.e. excluding permutations between coordinates; is expressed by the ratio, $N_{\mathrm{C}}=N_{\mathrm{C}}^{\prime} / N^{\prime}$, as:

$$
N_{\mathrm{C}}=\frac{11 !}{[11-(n-1)] !} \frac{1}{(n-1) !}=\frac{n}{12} \frac{12 !}{(12-n) ! n !}=\frac{n}{12}\left(\begin{array}{c}
12 \\
n
\end{array}\right)
$$

in terms of the binomial coefficients:

$$
\left(\begin{array}{l}
N \\
K
\end{array}\right)=\frac{N !}{K !(N-K) !}=\frac{N !}{(N-K) ! K !}=\left(\begin{array}{c}
N \\
N-K
\end{array}\right) ;
$$

related to any pair of natural numbers, $N, K, N \geq K$.

Accordingly, $N_{\mathrm{C}}$ is the number of distinct $n$-chords of cardinality, $n$. On the other hand, the total number of pitch-class sets of cardinality, $n$, regardless of Eq. (1), reads $12 N_{\mathrm{C}} / n$ e.g., [15] §29. Owing to Eq. (66), the dependence of $N_{\mathrm{C}}$ on $n$ is symmetric, as shown in Table 1. The additional case, $n=0$, has been added for completing the symmetry and shall be considered below. Further symmetries are exhibited by the fractional number of distinct $n$-chords, $N_{\mathrm{C}} / n$, and the related integer part, $I_{\mathrm{C}}=\operatorname{Int}\left(N_{\mathrm{C}} / n\right)$, via Eq. (6).

More specifically, Eq. (5) via (6) takes the equivalent form:

$$
\frac{N_{\mathrm{C}}}{n}=\frac{1}{12}\left(\begin{array}{c}
12 \\
n
\end{array}\right)=\frac{1}{12}\left(\begin{array}{c}
12 \\
12-n
\end{array}\right) ;
$$

and the related integer part is:

$$
I_{\mathrm{C}}=\operatorname{Int}\left(\frac{N_{\mathrm{C}}}{n}\right)=\operatorname{Int}\left[\frac{1}{12}\left(\begin{array}{c}
12 \\
n
\end{array}\right)\right]=\operatorname{Int}\left[\frac{1}{12}\left(\begin{array}{c}
12 \\
12-n
\end{array}\right)\right]
$$


Table 1: Number and fractional number of distinct $n$-chords, $N_{\mathrm{C}}, N_{\mathrm{C}} / n$, integer part, $I_{\mathrm{C}}=\operatorname{Int}\left(N_{\mathrm{C}} / n\right)$, number and fractional number of repeating $n$-chords, $\Delta N, \Delta N / n$, total number and fractional total number of $n$-chords, $N_{\mathrm{M}}=N_{\mathrm{C}}+\Delta N, N_{\mathrm{M}} / n$, for different cardinality, $n, 1 \leq n \leq 12$. The additional case, $n=0$, has been added for completing the symmetry. See text for further details.

\begin{tabular}{|c|c|c|c|c|c|c|c|c|c|c|c|c|c|}
\hline \hline$n$ & 0 & 1 & 2 & 3 & 4 & 5 & 6 & 7 & 8 & 9 & 10 & 11 & 12 \\
$N_{\mathrm{C}}$ & 0 & 1 & 11 & 55 & 165 & 330 & 462 & 462 & 330 & 165 & 55 & 11 & 1 \\
$\frac{N_{\mathrm{C}}}{n}$ & $\frac{1}{12}$ & 1 & $\frac{11}{2}$ & $\frac{55}{3}$ & $\frac{165}{4}$ & 66 & 77 & 66 & $\frac{165}{4}$ & $\frac{55}{3}$ & $\frac{11}{2}$ & 1 & $\frac{1}{12}$ \\
$I_{\mathrm{C}}$ & 0 & 1 & 5 & 18 & 41 & 66 & 77 & 66 & 41 & 18 & 5 & 1 & 0 \\
$\Delta N$ & 0 & 0 & 1 & 2 & 7 & 0 & 18 & 0 & 14 & 6 & 5 & 0 & 11 \\
$\frac{\Delta N}{n}$ & $\frac{11}{12}$ & 0 & $\frac{1}{2}$ & $\frac{2}{3}$ & $\frac{7}{4}$ & 0 & 3 & 0 & $\frac{7}{4}$ & $\frac{2}{3}$ & $\frac{1}{2}$ & 0 & $\frac{11}{12}$ \\
$N_{\mathrm{M}}$ & 0 & 1 & 12 & 57 & 172 & 330 & 480 & 462 & 344 & 171 & 60 & 11 & 12 \\
$\frac{N_{\mathrm{M}}}{n}$ & 1 & 1 & 6 & 19 & 43 & 66 & 80 & 66 & 43 & 19 & 6 & 1 & 1 \\
\hline
\end{tabular}

which is symmetric with respect to the maximum, occurring at $n=6$, as shown in Table 1 .

To complete the symmetry up to the extreme value, $n=12$, the domain must be extended down to the opposite extreme, $n=0$, conceived as representing the empty $n$-chord (no mode). To this aim, factorials must be expressed in terms of the Euler Gamma function e.g., [21] Chap. 16, as:

$$
\Gamma(n+1)=n \Gamma(n)=n ! ; \quad \Gamma(1)=1 ; \quad n=1,2,3, \ldots \quad ;
$$

where the recursion formula holds for all positive reals, in particular:

$$
\lim _{n \rightarrow 0^{+}}[n \Gamma(n)]=\lim _{n \rightarrow 0^{+}} \Gamma(n+1)=\Gamma(1)=1 ;
$$

or:

$$
\lim _{n \rightarrow 0^{+}} \Gamma(n)=\lim _{n \rightarrow 0^{+}} \frac{1}{n}
$$

and a similar result is found for $n \rightarrow 0^{-}$extending the recursion formula, Eq. (9), to the negative real semiaxis.

In terms of the Euler Gamma function, Eq. (7) reads:

$$
\frac{N_{\mathrm{C}}}{n}=\frac{1}{n} \frac{\Gamma(12)}{\Gamma(13-n) \Gamma(n)} ;
$$

which, for positive infinitesimal $n$, takes the expression:

$$
\lim _{n \rightarrow 0^{+}} \frac{N_{\mathrm{C}}}{n}=\lim _{n \rightarrow 0^{+}}\left[\frac{1}{n} \frac{\Gamma(12)}{\Gamma(13-n) \Gamma(n)}\right]=\frac{\Gamma(12)}{\Gamma(13)} \lim _{n \rightarrow 0^{+}}\left[\frac{1}{n} \frac{1}{\Gamma(n)}\right] ;
$$


and the combination of Eqs. (9), (10), (13), yields:

$$
\begin{aligned}
& \lim _{n \rightarrow 0^{+}} \frac{N_{\mathrm{C}}}{n}=\frac{11 !}{12 !}=\frac{1}{12} ; \\
& \lim _{n \rightarrow 0^{+}} \operatorname{Int}\left(\frac{N_{\mathrm{C}}}{n}\right)=0 ;
\end{aligned}
$$

which completes the symmetry of the fractional number, $N_{\mathrm{C}} / n$, and related integer part, $I_{\mathrm{C}}$, with respect to the maximum occurring at $n=6$. In authors' opinion, the above considerations add something more to the bare statement, that $0 !=1$ holds by definition.

With regard to a selected Euclidean $n$-space, an integer value of the fractional number, $N_{\mathrm{C}} / n$, makes a necessary (but not sufficient) condition for a one-to-one correspondence between projected points and coordinates, $\mathrm{Q}_{\mathrm{n}-1} \leftrightarrow\left\{s_{1}, s_{2}, \ldots, s_{n-1}\right\}$, where $s_{1}=12-\ell_{1} ; s_{k}=S_{k, n-1}, 2 \leq k \leq n-2$; $s_{n-1}=\ell_{n-1}$. An inspection of Table 1 shows the necessary condition fails in several cases, which implies the above mentioned correspondence is not one-to-one i.e. repeating coordinates, related to repeating $n$-chords, must also be enumerated.

\subsection{Enumeration of repeating $n$-chords}

With regard to a selected Euclidean $n$-space and a primitive form, $\mathrm{P}_{\mathrm{n}} \equiv$ $\left(\ell_{1}, \ell_{2}, \ldots, \ell_{n}\right)$, of an assigned $T_{n}$ set class, repeating (or transpositionally invariant) $n$-chords, $\mathrm{P}_{\mathrm{n}}^{\prime} \equiv\left(\ell_{1}^{\prime}, \ell_{2}^{\prime}, \ldots, \ell_{n}^{\prime}\right), \mathrm{P}_{\mathrm{n}}^{\prime \prime} \equiv\left(\ell_{1}^{\prime \prime}, \ell_{2}^{\prime \prime}, \ldots, \ell_{n}^{\prime \prime}\right)$, exhibit identical coordinates, $\ell_{j}^{\prime}=\ell_{j}^{\prime \prime}, 1 \leq j \leq n$. More specifically, a necessary condition for the occurrence of repeating $n$-chords is that the coordinates, $\left(\ell_{1}, \ell_{2}, \ldots, \ell_{n}\right)$, equal one to the other in the same number i.e. $\ell_{11}=\ell_{21}=\ldots=\ell_{i 1}$; $\ell_{12}=\ell_{22}=\ldots=\ell_{i 2} ; \ldots ; \ell_{1 k}=\ell_{2 k}=\ldots=\ell_{i k}$; where $1 \leq i k=n \leq 12$.

Accordingly, repeating $n$-chords exhibit identical soloes of coordinates, $\left(\ell_{1}, \ell_{1}, \ldots, \ell_{1}\right)$, or identical duoes of coordinates, $\left(\ell_{1}, \ell_{2}, \ell_{1}, \ell_{2}, \ldots, \ell_{1}, \ell_{2}\right)$, or identical trioes of coordinates, $\left(\ell_{1}, \ell_{2}, \ell_{3}, \ell_{1}, \ell_{2}, \ell_{3}, \ldots, \ell_{1}, \ell_{2}, \ell_{3}\right)$, or identical quartets of coordinates, $\left(\ell_{1}, \ell_{2}, \ell_{3}, \ell_{4}, \ell_{1}, \ell_{2}, \ell_{3}, \ell_{4}, \ell_{1}, \ell_{2}, \ell_{3}, \ell_{4}\right)$, or identical quintets of coordinates, $\left(\ell_{1}, \ell_{2}, \ell_{3}, \ell_{4}, \ell_{5}, \ell_{1}, \ell_{2}, \ell_{3}, \ell_{4}, \ell_{5}\right)$. Identical sextets are not considered in that they yield the chromatic scale, $\{1,1,1,1,1,1,1,1,1,1,1,1\}$, via Eq. (11), and thus reduce to identical soloes.

For repeating $n$-chords of cardinality, $n$, Eq. (1) reduces to:

$$
\ell_{1}+\ell_{2}+\ldots+\ell_{i}=\frac{12}{k}=\frac{12}{n} i
$$

where $i$ is the number of different coordinates and $k=n / i$ is their multiplicity. In any case, the number of repeating $n$-chords, $\Delta N_{i}(n)$, to be added 
to the number of distinct $n$-chords, $N_{\mathrm{C}}(n)$, has to be determined for $T_{n}$ set classes, while $T_{n} / T_{n} I$ set classes shall be considered afterwards.

Identical soloes of coordinates $(i=1, k=n)$ cannot occur for $n<2$, and Eq. (16) reduces to:

$$
\ell_{1}=\frac{12}{n} ; \quad n \geq 2 ;
$$

which implies the existence of $n$-chords with identical soloes of coordinates provided the ratio on the right-hand side of Eq. (17) is integer. The related $T_{n}$ set class is made of $n$ identical singletons of $n$-chords, one to be counted as distinct and the remaining $(n-1)$ to be added as repeating. Accordingly, the number of repeating $n$-chords reads:

$$
\Delta N_{1}(n)=\zeta(12, n)(n-1) \nu_{1}(n) ; \quad n \geq 2 ;
$$

where $\nu_{1}(n)$ is the number of $T_{n}$ set classes including $n$-chords which satisfy Eq. (17), more specifically $\nu_{1}(n)=1$ for $n=2,3,4,6,12$, and $\nu_{1}(n)=0$ otherwise. In general the function, $\zeta$, is defined as:

$$
\zeta\left(m_{1}, m_{2}\right)=\left\{\begin{array}{lll}
1 ; & \frac{m_{1}}{m_{2}}-\operatorname{Int}\left(\frac{m_{1}}{m_{2}}\right)=0 ; \\
1 ; & m_{2}=0 ; \\
0 ; & \frac{m_{1}}{m_{2}}-\operatorname{Int}\left(\frac{m_{1}}{m_{2}}\right)>0 ;
\end{array}\right.
$$

where $\operatorname{Int}(x)$ is the integer part of $x$ and $m_{1}, m_{2}, m_{1} \geq m_{2}$, are natural numbers, $m_{1}=12, m_{2}=n$, in the case under discussion.

Identical duoes of coordinates $(i=2, k=n / 2)$ cannot occur for $n<4$, and Eq. (16) reduces to:

$$
\ell_{1}+\ell_{2}=\frac{24}{n} ; \quad n \geq 4 ;
$$

which implies the existence of $n$-chords with identical duoes of coordinates provided the ratio on the right-hand side of Eq. (20) is integer. Related $T_{n}$ set classes are made of $n / 2$ identical doublets of $n$-chords, each one to be counted as distinct and the others to be added as repeating. Accordingly, the number of repeating $n$-chords reads:

$$
\Delta N_{2}(n)=\zeta(24, n)(n-2) \nu_{2}(n) ; \quad n \geq 4 ;
$$

where $\nu_{2}(n)$ is the number of $T_{n}$ set classes including $n$-chords which satisfy Eq. (20), to be determined for $n=4,6,8$, as $\nu_{2}(n)=0$ otherwise.

For $n=4$, Eq. (20) reduces to $\ell_{1}+\ell_{2}=6$ which has distinct (i.e. at least one different from the other) solutions as $\left(\ell_{1}, \ell_{2}\right)=(1,5),(2,4)$, implying $\nu_{2}(4)=1+1=2, \Delta N_{2}(4)=2+2=4$. 
For $n=6$, Eq. (201) reduces to $\ell_{1}+\ell_{2}=4$ which has distinct solutions as $\left(\ell_{1}, \ell_{2}\right)=(1,3)$, implying $\nu_{2}(6)=1, \Delta N_{2}(6)=2+2=4$.

For $n=8$, Eq. (20) reduces to $\ell_{1}+\ell_{2}=3$ which has distinct solutions as $\left(\ell_{1}, \ell_{2}\right)=(1,2)$, implying $\nu_{2}(8)=1, \Delta N_{2}(8)=3+3=6$. The 8-chord, $\{1,2,1,2,1,2,1,2\}$, is quoted among modes à transpositions limitées [12].

Identical trioes of coordinates $(i=3, k=n / 3)$ cannot occur for $n<6$, and Eq. (16) reduces to:

$$
\ell_{1}+\ell_{2}+\ell_{3}=\frac{36}{n} ; \quad n \geq 6 ;
$$

which implies the existence of $n$-chords with identical trioes of coordinates provided the ratio on the right-hand side of Eq. (22) is integer. Related $T_{n}$ set classes are made of $n / 3$ identical triplets of $n$-chords, each one to be counted as distinct and the others to be added as repeating. Accordingly, the number of repeating $n$-chords reads:

$$
\Delta N_{3}(n)=\zeta(36, n)(n-3) \nu_{3}(n) ; \quad n \geq 6 ;
$$

where $\nu_{3}(n)$ is the number of $T_{n}$ set classes including $n$-chords which satisfy Eq. (22), to be determined for $n=6,9$, as $\nu_{3}(n)=0$ otherwise.

For $n=6$, Eq. (22) reduces to $\ell_{1}+\ell_{2}+\ell_{3}=6$ which has distinct solutions as $\left(\ell_{1}, \ell_{2}, \ell_{3}\right)=(1,2,3),(1,1,4)$, implying $\nu_{3}(6)=2+1=3, \Delta N_{3}(6)=3+3+$ $3=9$. The 6 -chord, $\{1,4,1,1,4,1\}$, is quoted among modes à transpositions limitées [12].

For $n=9$, Eq. (22) reduces to $\ell_{1}+\ell_{2}+\ell_{3}=4$ which has distinct solutions as $\left(\ell_{1}, \ell_{2}, \ell_{3}\right)=(1,1,2)$, implying $\nu_{3}(9)=1, \Delta N_{3}(9)=2+2+2=6$. The 9 chord, $\{2,1,1,2,1,1,2,1,1\}$, is quoted among modes à transpositions limitées [12].

Identical quartets of coordinates $(i=4, k=n / 4)$ cannot occur for $n<8$, and Eq. (16) reduces to:

$$
\ell_{1}+\ell_{2}+\ell_{3}+\ell_{4}=\frac{48}{n} ; \quad n \geq 8 ;
$$

which implies the existence of $n$-chords with identical quartets of coordinates provided the ratio on the right-hand side of Eq. (24) is integer. Related $T_{n}$ set classes are made of $n / 4$ identical quadruplets of $n$-chords, each one to be counted as distinct and the others to be added as repeating. Accordingly, the number of repeating $n$-chords reads:

$$
\Delta N_{4}(n)=\zeta(48, n)(n-4) \nu_{4}(n) ; \quad n \geq 8 ;
$$

where $\nu_{4}(n)$ is the number of $T_{n}$ set classes including $n$-chords which satisfy Eq. (24), to be determined for $n=8$, as $\nu_{4}(n)=0$ otherwise. 
For $n=8$, Eq. (24) reduces to $\ell_{1}+\ell_{2}+\ell_{3}+\ell_{4}=6$ which has distinct solutions as $\left(\ell_{1}, \ell_{2}, \ell_{3}, \ell_{4}\right)=(1,1,2,2),(1,1,1,3)$, implying $\nu_{4}(8)=1+1=2$, $\Delta N_{4}(8)=4+4=8$. The 8 -chords, $\{2,2,1,1,2,2,1,1\},\{1,1,3,1,1,1,3,1\}$, are quoted among modes à transpositions limitées [12].

Identical quintets of coordinates $(i=5, k=n / 5)$ cannot occur for $n<10$, and Eq. (16) reduces to:

$$
\ell_{1}+\ell_{2}+\ell_{3}+\ell_{4}+\ell_{5}=\frac{60}{n} ; \quad n \geq 10 ;
$$

which implies the existence of $n$-chords with identical quintets of coordinates provided the ratio on the right-hand side of Eq. (26) is integer. Related $T_{n}$ set classes are made of $n / 5$ identical quintuplets of $n$-chords, each one to be counted as distinct and the others to be added as repeating. Accordingly, the number of repeating $n$-chords reads:

$$
\Delta N_{5}(n)=\zeta(60, n)(n-5) \nu_{5}(n) ; \quad n \geq 10 ;
$$

where $\nu_{5}(n)$ is the number of $T_{n}$ set classes including $n$-chords which satisfy Eq. (26), to be determined for $n=10$, as $\nu_{5}(n)=0$ otherwise.

For $n=10$, Eq. (26) reduces to $\ell_{1}+\ell_{2}+\ell_{3}+\ell_{4}+\ell_{5}=6$ which has distinct solutions as $\left(\ell_{1}, \ell_{2}, \ell_{3}, \ell_{4}, \ell_{5}\right)=(1,1,1,1,2)$, implying $\nu_{5}(10)=1$, $\Delta N_{5}(10)=5$. The 10-chord, $\{1,1,1,2,1,1,1,1,2,1\}$, is quoted among modes à transpositions limitées [12].

In summary, the number of repeating $n$-chords of each cardinality, with regard to $T_{n}$ set classes, reads:

$$
\Delta N(n)=\sum_{i=1}^{5} \Delta N_{i}(n)=\sum_{i=1}^{5} \zeta(12 i, n)(n-i) \nu_{i}(n)
$$

where the number of repeating $n$-chords including identical soloes $(i=1)$, $\Delta N_{1}$, duoes $(i=2), \Delta N_{2}$, trioes $(i=3), \Delta N_{3}$, quartets $(i=4), \Delta N_{4}$, quintets $(i=5), \Delta N_{5}$, and the total, $\Delta N=\sum_{i} \Delta N_{i}$, are listed in Table 2 ,

Similarly, the number of $T_{n}$ set classes including repeating $n$-chords, $\nu_{i}$, the total number of $T_{n}$ set classes including repeating $n$-chords, $\nu=\sum_{i} \nu_{i}$, the number of $T_{n}$ set classes including only distinct $n$-chords, $\nu_{\mathrm{C}}$, and the total number of $T_{n}$ set classes, $\nu_{\mathrm{M}}=N_{\mathrm{M}} / n$, are listed in Table 3 ,

The number of distinct + repeating $n$-chords of each cardinality, with respect to $T_{n}$ set classes, via Eqs. (5) and (28) reads:

$$
N_{\mathrm{M}}(n)=N_{\mathrm{C}}(n)+\Delta N(n)=\frac{n}{12}\left(\begin{array}{c}
12 \\
n
\end{array}\right)+\sum_{i=1}^{5} \zeta(12 i, n)(n-i) \nu_{i}(n) ;
$$


Table 2: Number of repeating $n$-chords of each cardinality, $n$, including identical singletons, $\Delta N_{1}$; doublets, $\Delta N_{2}$; triplets, $\Delta N_{3}$; quadruplets, $\Delta N_{4}$; quintuplets, $\Delta N_{5}$; total number of repeating $n$-chords, $\Delta N=\sum_{i} \Delta N_{i}$; total number of distinct $n$-chords, $N_{\mathrm{C}}$; and total number of distinct + repeating $n$-chords, $N_{\mathrm{M}}=N_{\mathrm{C}}+\Delta N$. See text for further details.

\begin{tabular}{|c|r|r|r|r|r|r|r|r|r|r|r|r|r|}
\hline \hline$n$ & 0 & 1 & 2 & 3 & 4 & 5 & 6 & 7 & 8 & 9 & 10 & 11 & 12 \\
$\Delta N_{1}$ & 0 & 0 & 1 & 2 & 3 & 0 & 5 & 0 & 0 & 0 & 0 & 0 & 11 \\
$\Delta N_{2}$ & 0 & 0 & 0 & 0 & 4 & 0 & 4 & 0 & 6 & 0 & 0 & 0 & 0 \\
$\Delta N_{3}$ & 0 & 0 & 0 & 0 & 0 & 0 & 9 & 0 & 0 & 6 & 0 & 0 & 0 \\
$\Delta N_{4}$ & 0 & 0 & 0 & 0 & 0 & 0 & 0 & 0 & 8 & 0 & 0 & 0 & 0 \\
$\Delta N_{5}$ & 0 & 0 & 0 & 0 & 0 & 0 & 0 & 0 & 0 & 0 & 5 & 0 & 0 \\
$\Delta N$ & 0 & 0 & 1 & 2 & 7 & 0 & 18 & 0 & 14 & 6 & 5 & 0 & 11 \\
$N_{\mathrm{C}}$ & 0 & 1 & 11 & 55 & 165 & 330 & 462 & 462 & 330 & 165 & 55 & 11 & 1 \\
$N_{\mathrm{M}}$ & 0 & 1 & 12 & 57 & 172 & 330 & 480 & 462 & 344 & 171 & 60 & 11 & 12 \\
\hline
\end{tabular}

Table 3: Number of $T_{n}$ set classes including repeating $n$-chords of each cardinality, $n$, made of identical soloes, $\nu_{1}$; duoes, $\nu_{2}$; trioes, $\nu_{3}$; quartets, $\nu_{4}$; quintets, $\nu_{5}$; total number of $T_{n}$ set classes including repeating $n$-chords, $\nu=\sum_{i} \nu_{i}$; total number of $T_{n}$ set classes including only distinct $n$-chords, $\nu_{\mathrm{C}}$; and total number of $T_{n}$ set classes including distinct + repeating $n$-chords, $\nu_{\mathrm{M}}=\nu_{\mathrm{C}}+\nu$. The $T_{0}$ set class has been arbitrarily conceived as including 0 -chords made of (no) identical soloes, to preserve symmetry in $\nu_{\mathrm{C}}$ and $\nu$. See text for further details.

\begin{tabular}{|c|r|r|r|r|r|r|r|r|r|r|r|r|r|}
\hline \hline$n$ & 0 & 1 & 2 & 3 & 4 & 5 & 6 & 7 & 8 & 9 & 10 & 11 & 12 \\
$\nu_{1}$ & 1 & 0 & 1 & 1 & 1 & 0 & 1 & 0 & 0 & 0 & 0 & 0 & 1 \\
$\nu_{2}$ & 0 & 0 & 0 & 0 & 2 & 0 & 1 & 0 & 1 & 0 & 0 & 0 & 0 \\
$\nu_{3}$ & 0 & 0 & 0 & 0 & 0 & 0 & 3 & 0 & 0 & 1 & 0 & 0 & 0 \\
$\nu_{4}$ & 0 & 0 & 0 & 0 & 0 & 0 & 0 & 0 & 2 & 0 & 0 & 0 & 0 \\
$\nu_{5}$ & 0 & 0 & 0 & 0 & 0 & 0 & 0 & 0 & 0 & 0 & 1 & 0 & 0 \\
$\nu$ & 1 & 0 & 1 & 1 & 3 & 0 & 5 & 0 & 3 & 1 & 1 & 0 & 1 \\
$\nu_{\mathrm{C}}$ & 0 & 1 & 5 & 18 & 40 & 66 & 75 & 66 & 40 & 18 & 5 & 1 & 0 \\
$\nu_{\mathrm{M}}$ & 1 & 1 & 6 & 19 & 43 & 66 & 80 & 66 & 43 & 19 & 6 & 1 & 1 \\
\hline
\end{tabular}


and the number of $T_{n}$ set classes of each cardinality is:

$\nu_{\mathrm{M}}(n)=\frac{N_{\mathrm{M}}(n)}{n}=\frac{N_{\mathrm{C}}(n)+\Delta N(n)}{n}=\frac{1}{12}\left(\begin{array}{c}12 \\ n\end{array}\right)+\frac{1}{n} \sum_{i=1}^{5} \zeta(12 i, n)(n-i) \nu_{i}(n)$

where an inspection of Table 1 shows that, in general, the number of $T_{n}$ set classes including only distinct or repeating $n$-chords is different from $N_{\mathrm{C}}(n) / n$ or $\Delta N(n) / n$, respectively.

The above results complete the calculation of $\Delta N, \Delta N / n$, within the domain, $1 \leq n \leq 12$, which allows the knowledge of the total number of (distinct + repeating) $n$-chords, $N_{\mathrm{M}}=N_{\mathrm{C}}+\Delta N$, and the total number of $T_{n}$ set classes, $\nu_{\mathrm{M}}$, which are also listed in Tables 1, 2, 3. It can be seen $T_{n}$ set classes are symmetric with respect to $n=6$, within the domain, $1 \leq n \leq 11$. The extension of the domain to $n=0$ can be made demanding symmetry with respect to $n=12$, which implies the following:

$$
\begin{aligned}
& \lim _{n \rightarrow 0^{+}} \frac{N_{\mathrm{M}}(n)}{n}=\frac{N_{\mathrm{M}}(12)}{12}=1 ; \\
& \lim _{n \rightarrow 0^{+}} \frac{\Delta N(n)}{n}=\lim _{n \rightarrow 0^{+}}\left(\frac{N_{\mathrm{M}}(n)}{n}-\frac{N_{\mathrm{C}}(n)}{n}\right)=\frac{N_{\mathrm{M}}(12)}{12}-\frac{N_{\mathrm{C}}(12)}{12}=\frac{11}{12}
\end{aligned}
$$

as shown in Table 1.

Following a similar procedure, the number of $T_{n} / T_{n} I$ set classes of each cardinality can also be determined. In this view, for instance, the $T_{n} / T_{n} I$ set class,

$$
\begin{aligned}
& \{1,2,3,6\},\{2,3,6,1\},\{3,6,1,2\},\{6,1,2,3\}, \\
& \{6,3,2,1\},\{1,6,3,2\},\{2,1,6,3\},\{3,2,1,6\} ;
\end{aligned}
$$

is made of eight distinct 4-chords, while the $T_{n} / T_{n} I$ set class,

$$
\begin{aligned}
& \{1,5,5,1\},\{5,5,1,1\},\{5,1,1,5\},\{1,1,5,5\}, \\
& \{1,5,5,1\},\{1,1,5,5\},\{5,1,1,5\},\{5,5,1,1\} ;
\end{aligned}
$$

is made of four distinct and four repeating 4-chords.

With regard to a $n$-chord of cardinality, $n$, let the $n$-chord type be defined as $\ell_{1}^{i_{1}} \ell_{2}^{i_{2}} \ldots \ell_{k}^{i_{k}}$, where $i_{j}$ denotes the multiplicity of the coordinate, $\ell_{j}, 1 \leq$ $j \leq k$, which implies $i_{1}+i_{2}+\ldots+i_{k}=n$. Clearly the enumeration of distinct $n$-chords remains unchanged and the results valid for $T_{n}$ set classes maintain for $T_{n} / T_{n} I$ set classes. Conversely, the number of repeating $n$ chords is expected to grow due to larger cardinality, $2 n$, of $T_{n} / T_{n} I$ set classes, which implies additional kind of repeating $n$-chords with respect to $T_{n}$ set 
classes i.e. $n$-chords made of identical singletons, duoes, trioes, quartets, quintets of coordinates.

In this view, let palindrome $n$-chords be defined as invariant with respect to reflection, and pseudo palindrome $n$-chords as invariant with respect to reflection after appropriate transposition. For instance, $\{1,5,5,1\}$ is palindrome while $\{5,5,1,1\}$ is pseudo palindrome. Palindrome and pseudo palindrome $n$-chords make the additional kinds of repeating $n$-chords, in connection with $T_{n} / T_{n} I$ set classes. Then the number of repeating palindrome and pseudo palindrome $n$-chords of each cardinality, which have not previously considered, has to be determined.

With regard to $T_{n} / T_{n} I$ set classes of each cardinality from 1 to 12 (with the addition of 0 ), equivalence classes are made of $2 n n$-chords which are related via circular permutation and reflection. The total number can be determined along the following steps.

(i) Start from $T_{n}$ set classes of each cardinality.

(ii) Separate $T_{n}$ set classes exhibiting neither palindrome nor pseudo palindrome $n$-chords, let the total number be denoted as $\nu_{\mathrm{N}}(n)$, from $T_{n}$ set classes exhibiting palindrome or pseudo palindrome $n$-chords, let the total number be denoted as $\nu_{\mathrm{P}}(n)$.

(iii) Determine $\nu_{\mathrm{N}}(n)$ and $\nu_{\mathrm{P}}(n)$.

(iv) Calculate the total number of $T_{n} / T_{n} I$ set classes as $\nu_{\mathrm{Q}}(n)=\nu_{\mathrm{N}}(n) / 2+$ $\nu_{\mathrm{P}}(n)$, according to the above considerations.

Palindrome and pseudo palindrome $n$-chords are necessarily made of pairs of identical coordinates e.g., $\{1,2,3,3,2,1\}$, for even cardinality, with the addition of a single coordinate e.g., $\{1,4,2,4,1\}$, for odd cardinality. Then Eq. (11) reduces to:

$$
\begin{aligned}
& k_{1} \ell_{1}+k_{2} \ell_{2}+\ldots+k_{i} \ell_{i}=12 ; \\
& k_{1} \ell_{1}+k_{2} \ell_{2}+\ldots+k_{i} \ell_{i}+\ell_{i+1}=12 ;
\end{aligned}
$$

respectively, where $k_{j}, 1 \leq j \leq i$, is the multiplicity of the coordinate, $\ell_{j}$. Let $T_{n}$ set classes made of palindrome and pseudo palindrome $n$-chords be denoted as $T_{n, \mathrm{P}}$.

For $n=0,1, n$-chords remain unchanged after transposition and/or reflection. For $n=2$, transposition and reflection are equivalent or, in other words, all $n$-chords are palindrome or pseudo palindrome. Accordingly, $\nu_{\mathrm{Q}}(n)=\nu_{\mathrm{M}}(n), n<3$, where the number of $T_{n}$ set classes, $\nu_{\mathrm{M}}(n)$, is listed in Table 3 , 
For $n=3$, Eq. (33b) reduces to:

$$
2 \ell_{1}+\ell_{2}=12 ; \quad n=3 ;
$$

which has solutions as $\left(\ell_{1}, \ell_{2}\right)=(1,10),(2,8),(3,6),(4,4),(5,2)$, implying $\nu_{\mathrm{P}}(3)=5, \nu_{\mathrm{N}}(3)=\nu_{\mathrm{M}}(3)-\nu_{\mathrm{P}}(3)=19-5=14 ; \nu_{\mathrm{Q}}(3)=\nu_{\mathrm{N}}(3) / 2+\nu_{\mathrm{P}}(3)=$ $14 / 2+5=7+5=12$.

For $n=4$, Eq. (33a) reduces to:

$$
\begin{aligned}
& 2 \ell_{1}+\ell_{2}+\ell_{3}=12 ; \quad n=4 ; \\
& 2 \ell_{1}+2 \ell_{2}=12 ; \quad n=4 ; \\
& 3 \ell_{1}+\ell_{2}=12 ; \quad n=4 ;
\end{aligned}
$$

which has solutions as $\left(\ell_{1}, \ell_{2}, \ell_{3}\right)=(1,2,8),(1,3,7),(1,4,6),(2,1,7),(2,3,5)$, $(3,1,5),(3,2,4),(4,1,3),(3,3,3) ;\left(\ell_{1}, \ell_{2}\right)=(1,5),(2,4) ;\left(\ell_{1}, \ell_{2}\right)=(1,9),(2,6)$; respectively.

Case a yields one kind of $T_{n, \mathrm{P}}$ set classes, namely $\{x, y, x, z\}$. Accordingly, $\nu_{\mathrm{Pa}}(4)=1 \cdot 9=9$.

Case b yields two kinds of $T_{n, \mathrm{P}}$ set classes, namely $\{x, x, y, y\},\{x, y, x, y\}$. Accordingly, $\nu_{\mathrm{Pb}}(4)=2 \cdot 2=4$.

Case c yields one kind of $T_{n, \mathrm{P}}$ set classes, namely $\{x, x, x, y\}$. Accordingly, $\nu_{\mathrm{Pc}}(4)=1 \cdot 2=2$.

Finally, $\nu_{\mathrm{P}}(4)=\sum_{i} \nu_{\mathrm{Pi}}(4)=9+4+2=15 ; \nu_{\mathrm{N}}(4)=\nu_{\mathrm{M}}(4)-\nu_{\mathrm{P}}(4)=$ $43-15=28 ; \nu_{\mathrm{Q}}(4)=\nu_{\mathrm{N}}(4) / 2+\nu_{\mathrm{P}}(4)=28 / 2+15=14+15=29$.

For $n=5$, Eq. (33b) reduces to:

$$
\begin{aligned}
& 4 \ell_{1}+\ell_{2}=12 ; \quad n=5 ; \\
& 2 \ell_{1}+2 \ell_{2}+\ell_{3}=12 ; \quad n=5 ; \\
& 3 \ell_{1}+2 \ell_{2}=12 ; \quad n=5 ;
\end{aligned} ;
$$

which has solutions as $\left(\ell_{1}, \ell_{2}\right)=(1,8),(2,4) ;\left(\ell_{1}, \ell_{2}, \ell_{3}\right)=(1,2,6),(1,3,4)$, $(1,4,2) ;\left(\ell_{1}, \ell_{2}\right)=(2,3)$; respectively.

Case a yields one kind of $T_{n, \mathrm{P}}$ set classes, namely $\{x, x, x, x, y\}$. Accordingly, $\nu_{\mathrm{Pa}}(5)=1 \cdot 2=2$.

Case b yields two kinds of $T_{n, \mathrm{P}}$ set classes, namely $\{x, y, z, y, x\},\{y, x, z, x, y\}$. Accordingly, $\nu_{\mathrm{Pb}}(5)=2 \cdot 3=6$.

Case c yields two kinds of $T_{n, \mathrm{P}}$ set classes, namely $\{x, x, x, y, y\},\{x, y, x, y, x\}$. Accordingly, $\nu_{\mathrm{Pc}}(5)=2 \cdot 1=2$.

Finally, $\nu_{\mathrm{P}}(5)=\sum_{i} \nu_{\mathrm{Pi}}(5)=2+6+2=10 ; \nu_{\mathrm{N}}(5)=\nu_{\mathrm{M}}(5)-\nu_{\mathrm{P}}(5)=$ $66-10=56 ; \nu_{\mathrm{Q}}(5)=\nu_{\mathrm{N}}(5) / 2+\nu_{\mathrm{P}}(5)=56 / 2+10=28+10=38$. 
For $n=6$, Eq. (33a) reduces to:

$$
\begin{aligned}
& 2 \ell_{1}+2 \ell_{2}+2 \ell_{3}=12 ; \quad n=6 ; \\
& 3 \ell_{1}+3 \ell_{2}=12 ; \quad n=6 ; \\
& 3 \ell_{1}+2 \ell_{2}+\ell_{3}=12 ; \quad n=6 ; \\
& 4 \ell_{1}+2 \ell_{2}=12 ; \quad n=6 ; \\
& 4 \ell_{1}+\ell_{2}+\ell_{3}=12 ; \quad n=6 ; \\
& 5 \ell_{1}+\ell_{2}=12 ; \quad n=6 ;
\end{aligned}
$$

which has solutions as $\left(\ell_{1}, \ell_{2}, \ell_{3}\right)=(1,2,3) ;\left(\ell_{1}, \ell_{2}\right)=(1,3) ;\left(\ell_{1}, \ell_{2}, \ell_{3}\right)=$ $(1,2,5),(2,1,4) ;\left(\ell_{1}, \ell_{2}\right)=(1,4) ;\left(\ell_{1}, \ell_{2}, \ell_{3}\right)=(1,2,6),(1,3,5),(2,1,3) ;\left(\ell_{1}, \ell_{2}\right)=$ $(1,7),(2,2)$; respectively.

Case a yields six kinds of $T_{n, \mathrm{P}}$ set classes, namely $\{x, y, x, z, y, z\},\{x, z, x, y, z, y\}$, $\{y, x, y, z, x, z\},\{x, z, y, y, z, x\},\{y, x, z, z, x, y\},\{z, y, x, x, y, z\}$. Accordingly, $\nu_{\mathrm{Pa}}(6)=6 \cdot 1=6$.

Case b yields two kinds of $T_{n, \mathrm{P}}$ set classes, namely $\{x, x, x, y, y, y\},\{x, y, x, y, x, y\}$. Accordingly, $\nu_{\mathrm{Pb}}(6)=2 \cdot 1=2$.

Case c yields two kinds of $T_{n, \mathrm{P}}$ set classes, namely $\{x, x, x, y, z, y\},\{x, z, x, y, x, y\}$. Accordingly, $\nu_{\mathrm{Pc}}(6)=2 \cdot 2=4$.

Case $\mathrm{d}$ yields three kinds of $T_{n, \mathrm{P}}$ set classes, namely $\{x, x, y, y, x, x\}$, $\{x, y, x, x, y, x\},\{x, x, x, y, x, y\}$. Accordingly, $\nu_{\mathrm{Pd}}(6)=3 \cdot 1=3$.

Case e yields one kind of $T_{n, \mathrm{P}}$ set classes, namely $\{x, y, x, x, z, x\}$. Accordingly, $\nu_{\mathrm{Pe}}(6)=1 \cdot 3=3$.

Case $\mathrm{f}$ yields one kind of $T_{n, \mathrm{P}}$ set classes, namely $\{x, x, x, x, x, y\}$. Accordingly, $\nu_{\mathrm{Pf}}(6)=1 \cdot 2=2$.

Finally, $\nu_{\mathrm{P}}(6)=\sum_{i} \nu_{\mathrm{Pi}}(6)=6+2+4+3+3+2=20 ; \nu_{\mathrm{N}}(6)=\nu_{\mathrm{M}}(6)-$ $\nu_{\mathrm{P}}(6)=80-20=60 ; \nu_{\mathrm{Q}}(6)=\nu_{\mathrm{N}}(6) / 2+\nu_{\mathrm{P}}(6)=60 / 2+20=30+20=50$.

For $n=7$, Eq. (33b) reduces to:

$$
\begin{aligned}
& 6 \ell_{1}+\ell_{2}=12 ; \quad n=7 ; \\
& 4 \ell_{1}+2 \ell_{2}+\ell_{3}=12 ; \quad n=7 ; \\
& 5 \ell_{1}+2 \ell_{2}=12 ; \quad n=7 ;
\end{aligned}
$$

which has solutions as $\left(\ell_{1}, \ell_{2}\right)=(1,6) ;\left(\ell_{1}, \ell_{2}, \ell_{3}\right)=(1,2,4),(1,3,2) ;\left(\ell_{1}, \ell_{2}\right)=$ $(2,1)$; respectively.

Case a yields one kind of $T_{n, \mathrm{P}}$ set classes, namely $\{x, x, x, x, x, x, y\}$. Accordingly, $\nu_{\mathrm{Pa}}(7)=1 \cdot 1=1$.

Case $\mathrm{b}$ yields three kinds of $T_{n, \mathrm{P}}$ set classes, namely $\{x, x, y, z, y, x, x\}$, $\{x, y, x, z, x, y, x\},\{y, x, x, z, x, x, y\}$. Accordingly, $\nu_{\mathrm{Pb}}(7)=3 \cdot 2=6$.

Case c yields three kinds of $T_{n, \mathrm{P}}$ set classes, namely $\{y, x, x, x, x, x, y\}$, $\{x, y, x, x, y, x\},\{x, x, y, x, y, x, x\}$. Accordingly, $\nu_{\mathrm{Pc}}(7)=3 \cdot 1=3$. 
Finally, $\nu_{\mathrm{P}}(7)=\sum_{i} \nu_{\mathrm{Pi}}(7)=1+6+3=10 ; \nu_{\mathrm{N}}(7)=\nu_{\mathrm{M}}(7)-\nu_{\mathrm{P}}(7)=$ $66-10=56 ; \nu_{\mathrm{Q}}(7)=\nu_{\mathrm{N}}(7) / 2+\nu_{\mathrm{P}}(7)=56 / 2+10=28+10=38$.

For $n=8$, Eq. (33a) reduces to:

$$
\begin{aligned}
& 4 \ell_{1}+4 \ell_{2}=12 ; \quad n=8 ; \\
& 6 \ell_{1}+\ell_{2}+\ell_{3}=12 ; \quad n=8 ; \\
& 5 \ell_{1}+2 \ell_{2}+\ell_{3}=12 ; \quad n=8 ; \\
& 6 \ell_{1}+2 \ell_{2}=12 ; \quad n=8 ; \\
& 7 \ell_{1}+\ell_{2}=12 ; \quad n=8 ;
\end{aligned}
$$

which has solutions as $\left(\ell_{1}, \ell_{2}\right)=(1,2) ;\left(\ell_{1}, \ell_{2}, \ell_{3}\right)=(1,2,4) ;\left(\ell_{1}, \ell_{2}, \ell_{3}\right)=$ $(1,2,3) ;\left(\ell_{1}, \ell_{2}\right)=(1,3) ;\left(\ell_{1}, \ell_{2}\right)=(1,5)$; respectively.

Case a yields six kinds of $T_{n, \mathrm{P}}$ set classes, namely $\{x, x, x, x, y, y, y, y\}$, $\{x, x, y, y, x, x, y, y\},\{x, y, x, y, x, y, x, y\},\{x, y, x, y, y, x, y, x\},\{x, x, y, y, x, y, y, x\}$, $\{x, x, y, x, x, y, y, y\}$. Accordingly, $\nu_{\mathrm{Pa}}(8)=6 \cdot 1=6$.

Case $\mathrm{b}$ yields one kind of $T_{n, \mathrm{P}}$ set classes, namely $\{x, x, x, y, x, x, x, z\}$. Accordingly, $\nu_{\mathrm{Pb}}(8)=1 \cdot 1=1$.

Case $\mathrm{c}$ yields three kinds of $T_{n, \mathrm{P}}$ set classes, namely $\{x, x, x, y, z, y, x, x\}$, $\{x, x, y, x, z, x, y, x\},\{x, y, x, x, z, x, x, y\}$. Accordingly, $\nu_{\mathrm{Pc}}(8)=3 \cdot 1=3$.

Case d yields four kinds of $T_{n, \mathrm{P}}$ set classes, namely $\{x, x, x, x, x, x, y, y\}$, $\{x, x, x, x, x, y, x, y\},\{x, x, x, x, y, x, x, y\},\{x, x, x, y, x, x, x, y\}$. Accordingly, $\nu_{\mathrm{Pd}}(8)=4 \cdot 1=4$.

Case e yields one kind of $T_{n, \mathrm{P}}$ set classes, namely $\{x, x, x, x, x, x, x, y\}$. Accordingly, $\nu_{\mathrm{Pe}}(8)=1 \cdot 1=1$.

Finally, $\nu_{\mathrm{P}}(8)=\sum_{i} \nu_{\mathrm{Pi}}(8)=6+1+3+4+1=15 ; \nu_{\mathrm{N}}(8)=\nu_{\mathrm{M}}(8)-\nu_{\mathrm{P}}(8)=$ $43-15=28 ; \nu_{\mathrm{Q}}(8)=\nu_{\mathrm{N}}(8) / 2+\nu_{\mathrm{P}}(8)=28 / 2+15=14+15=29$.

For $n=9$, Eq. (33b) reduces to:

$$
\begin{aligned}
& 8 \ell_{1}+\ell_{2}=12 ; \quad n=9 ; \\
& 6 \ell_{1}+3 \ell_{2}=12 ; \quad n=9 ;
\end{aligned}
$$

which has solutions as $\left(\ell_{1}, \ell_{2}\right)=(1,4) ;\left(\ell_{1}, \ell_{2}\right)=(1,2)$; respectively.

Case a yields one kind of $T_{n, \mathrm{P}}$ set classes, namely $\{x, x, x, x, x, x, x, x, y\}$. Accordingly, $\nu_{\mathrm{Pa}}(9)=1 \cdot 1=1$.

Case b yields four kinds of $T_{n, \mathrm{P}}$ set classes, namely $\{x, x, x, y, y, y, x, x, x\}$, $\{x, x, y, x, y, x, y, x, x\},\{x, y, x, x, y, x, x, y, x\},\{y, x, x, x, y, x, x, x, y\}$. Accordingly, $\nu_{\mathrm{Pb}}(9)=4 \cdot 1=4$.

Finally, $\nu_{\mathrm{P}}(9)=\sum_{i} \nu_{\mathrm{Pi}}(9)=1+4=5 ; \nu_{\mathrm{N}}(9)=\nu_{\mathrm{M}}(9)-\nu_{\mathrm{P}}(9)=19-5=$ $14 ; \nu_{\mathrm{Q}}(9)=\nu_{\mathrm{N}}(9) / 2+\nu_{\mathrm{P}}(9)=14 / 2+5=7+5=12$.

For $n=10$, Eq. (33a) reduces to:

$$
\begin{aligned}
& 8 \ell_{1}+2 \ell_{2}=12 ; \quad n=10 ; \\
& 9 \ell_{1}+\ell_{2}=12 ; \quad n=10 ;
\end{aligned}
$$


Table 4: Number of $T_{n}$ set classes, $\nu_{\mathrm{M}}(n)$, palindrome and pseudo palindrome $T_{n}$ set classes, $\nu_{\mathrm{P}}(n)$, neither palindrome nor pseudo palindrome $T_{n}$ set classes, $\nu_{\mathrm{N}}(n)=\nu_{\mathrm{M}}(n)-\nu_{\mathrm{P}}(n)$, and $T_{n} / T_{n} I$ set classes, $\nu_{\mathrm{Q}}(n)=$ $\nu_{\mathrm{N}}(n) / 2+\nu_{\mathrm{P}}(n)$, involving $n$-chords of each cardinality, $n, 0 \leq n \leq 12$. See text for further details.

\begin{tabular}{|c|r|r|r|r|r|r|r|r|r|r|r|r|r|}
\hline \hline$n$ & 0 & 1 & 2 & 3 & 4 & 5 & 6 & 7 & 8 & 9 & 10 & 11 & 12 \\
$\nu_{\mathrm{M}}$ & 1 & 1 & 6 & 19 & 43 & 66 & 80 & 66 & 43 & 19 & 6 & 1 & 1 \\
$\nu_{\mathrm{P}}$ & 1 & 1 & 6 & 5 & 15 & 10 & 20 & 10 & 15 & 5 & 6 & 1 & 1 \\
$\nu_{\mathrm{N}}$ & 0 & 0 & 0 & 14 & 28 & 56 & 60 & 56 & 28 & 14 & 0 & 0 & 0 \\
$\nu_{\mathrm{Q}}$ & 1 & 1 & 6 & 12 & 29 & 38 & 50 & 38 & 29 & 12 & 6 & 1 & 1 \\
\hline
\end{tabular}

which has solutions as $\left(\ell_{1}, \ell_{2}\right)=(1,2) ;\left(\ell_{1}, \ell_{2}\right)=(1,4)$; respectively.

Case a yields five kinds of $T_{n, \mathrm{P}}$ set classes, namely $\{x, x, x, x, x, x, x, x, y, y\}$, $\{x, x, x, x, x, x, x, y, x, y\},\{x, x, x, x, x, x, y, x, x, y\},\{x, x, x, x, x, y, x, x, x, y\}$, $\{x, x, x, x, y, x, x, x, x, y\}$. Accordingly, $\nu_{\mathrm{Pa}}(10)=5 \cdot 1=5$.

Case b yields one kind of $T_{n, \mathrm{P}}$ set classes, namely $\{x, x, x, x, x, x, x, x, x, y\}$. Accordingly, $\nu_{\mathrm{Pb}}(10)=1 \cdot 1=1$.

Finally, $\nu_{\mathrm{P}}(10)=\sum_{i} \nu_{\mathrm{Pi}}(10)=5+1=6 ; \nu_{\mathrm{N}}(10)=\nu_{\mathrm{M}}(10)-\nu_{\mathrm{P}}(10)=$ $6-6=0 ; \nu_{\mathrm{Q}}(10)=\nu_{\mathrm{N}}(10) / 2+\nu_{\mathrm{P}}(10)=0 / 2+6=0+6=6$.

For $n=11$, Eq. (33b) reduces to:

$$
10 \ell_{1}+\ell_{2}=12 ; \quad n=11 ;
$$

which has solutions as $\left(\ell_{1}, \ell_{2}\right)=(1,2)$, yielding one kind of $T_{n, \mathrm{P}}$ set classes, namely $\{x, x, x, x, x, x, x, x, x, x, y\}$. Accordingly, $\nu_{\mathrm{P}}(11)=1 \cdot 1=1 ; \nu_{\mathrm{N}}(11)=$ $\nu_{\mathrm{M}}(11)-\nu_{\mathrm{P}}(11)=1-1=0 ; \nu_{\mathrm{Q}}(11)=\nu_{\mathrm{N}}(11) / 2+\nu_{\mathrm{P}}(11)=0 / 2+1=0+1=1$.

For $n=12$, Eq. (33a) reduces to:

$$
12 \ell_{1}=12 ; \quad n=12 ;
$$

which has solutions as $\ell_{1}=1$, yielding one kind of $T_{n, \mathrm{P}}$ set classes, namely $\{x, x, x, x, x, x, x, x, x, x, x, x\}$. Accordingly, $\nu_{\mathrm{P}}(12)=1 \cdot 1=1 ; \nu_{\mathrm{N}}(12)=$ $\nu_{\mathrm{M}}(12)-\nu_{\mathrm{P}}(12)=1-1=0 ; \nu_{\mathrm{Q}}(12)=\nu_{\mathrm{N}}(12) / 2+\nu_{\mathrm{P}}(12)=0 / 2+1=0+1=1$.

The total number of $T_{n}$ set classes, $\nu_{\mathrm{M}}(n)$ (listed in Table 3 and repeated for better comparison), palindrome and pseudo palindrome $T_{n}$ set classes, $\nu_{\mathrm{P}}(n)$, neither palindrome nor pseudo palindrome $T_{n}$ set classes, $\nu_{\mathrm{N}}(n)=$ $\nu_{\mathrm{M}}(n)-\nu_{\mathrm{P}}(n)$, and $T_{n} / T_{n} I$ set classes, $\nu_{\mathrm{Q}}(n)=\nu_{\mathrm{N}}(n) / 2+\nu_{\mathrm{P}}(n)$, are listed in Table 4 .

An inspection of Table 4 shows a symmetry with respect to $n=6$. This is why complementation gives a one to one correspondence between $T_{n}$ set 
classes of cardinality, $n$ and $12-n$, respectively, which is preserved for $T_{n} / T_{n} I$ set classes e.g., 14] Chap. $9 \S 9.14$. The same kind of symmetry is also implicit in the binomial formula, expressed by Eq. (6) .

\section{Geometrical interpretation}

With regard to an Euclidean $n$-space, $\Re^{n}$, and a Cartesian orthogonal reference frame, $\left(\mathrm{O} x_{1} x_{2}, \ldots x_{n}\right)$, the extension of the boundary condition, expressed by Eq. (1), to real numbers, reads:

$$
x_{1}+x_{2}+\ldots+x_{n}=12 ; 1 \leq n \leq 12 ;
$$

which represents a $(n-1)$-plane intersecting the coordinate axes at the points, $\mathrm{V}_{\mathrm{k}} \equiv\left(12 \delta_{1 k}, 12 \delta_{2 k}, \ldots, 12 \delta_{n k}\right), 1 \leq k \leq n$, where $\delta_{i k}$ is the Kronecker symbol.

The following properties can be established: (i) the $(n-1)$-plane, expressed by Eq. (44), is normal to the $n$-sector ( $n=2$, bisector; $n=3$, trisector; and so on) of the positive $2^{n}$-ant; (ii) the region of $(n-1)$-plane, bounded by the positive $2^{n}$-ant, is a regular, inclined $n$-hedron, $\Psi_{12}^{n}$, of vertexes, $\mathrm{V}_{\mathrm{k}}$, $1 \leq k \leq n$; (iii) special cases are $\Psi_{12}^{1}$, regular vertex; $\Psi_{12}^{2}$, regualr side; $\Psi_{12}^{3}$, regular triangle; $\Psi_{12}^{4}$, regular tetrahedron; (iv) the orthocentre of $\Psi_{12}^{n}$, $\mathrm{H}_{\mathrm{n}} \equiv(12 / n, 12 / n, \ldots, 12 / n)$, is the intersection between the $(n-1)$-plane and the $n$-sector of the positive $2^{n}$-ant. For further details, an interested reader is addressed to Appendix C.

In general, $\Psi_{12}^{n}$ may be divided into $n$ congruent $n$-hedrons, $\Psi_{12, i}^{n}, 1 \leq$ $i \leq n$, by joining the vertexes with the orthocentre. More specifically, the orthocentre is the common vertex while the remaining $(n-1)$ vertexes lie on a $(n-2)$ hyperface, or $(n-2)$-face, of $\Psi_{12}^{n}$.

The special case, $\Psi_{12}^{3}$, is represented in Fig.2 and related $n$-chords are shown as coordinates of positive integer points, satisfying the boundary condition expressed by Eq. (1), in Fig. 3.

According to the above considerations, $n$-chords are represented as coordinates of positive integer points within $\Psi_{12}^{n}$ i.e. satisfying the boundary condition expressed by Eq. (1). $T_{n}$ and $T_{n} / T_{n} I$ set classes contain $n$ and $n+n=2 n$ points of the kind considered, respectively, which implies (i) points with distinct coordinates, belonging to the same $T_{n}$ or $T_{n} / T_{n} I$ set class, are similarly placed within different $\Psi_{12, i}^{n}, 1 \leq i \leq n$; (ii) points with identical soloes, duoes, trioes, quartets, and quintets of coordinates are placed on $(n-2)$-faces between different $\Psi_{12, i}^{n}, i=i_{1}, i_{2}, 1 \leq i_{1}<i_{2} \leq n$; (iii) positive integer points (taking into due account the multiciplity of repeating $n$-chords) are equally partitioned among $\Psi_{12, i}^{n}, 1 \leq i \leq n$, in number 
of $\nu_{\mathrm{M}}(n)=N_{\mathrm{M}}(n) / n$ via Eq. (30). For complementary Euclidean $n$-spaces, $\Re^{n} \leftrightarrow \Re^{12-n}, \nu_{\mathrm{M}}(n)=\nu_{\mathrm{M}}(12-n)$ as shown in Table 3 .

In general, $\Psi_{12, i}^{n}, 1 \leq i \leq n$, have basis coinciding with the $i$ th $(n-2)$-face of $\Psi_{12}^{n}$, which implies $(n-1)$ vertexes in common, with the inclusion of the orthocentre of $\Psi_{12}^{n}$. Accordingly, the coordinates of a generic positive integer point, $\mathrm{P}_{\mathrm{i}} \equiv\left(\ell_{1}, \ell_{2}, \ldots, \ell_{n}\right)$, belonging to $\Psi_{12, i}^{n}, 1 \leq i \leq n$, have necessarily to satisfy the conditions:

$$
1 \leq \ell_{i} \leq \frac{12}{n} ; \quad 1 \leq \ell_{j} \leq 12-(n-1) ; \quad 1 \leq j \leq 12 ; \quad j \neq i
$$

where the vertex of $\Psi_{12}^{n}$, placed on the coordinate axis, $x_{i}$, does not belong to the congruent $n$-hedron under consideration. It is apparent circular permutation of coordinates makes the points, $\mathrm{P}_{\mathrm{i}}$, be similarly placed within different $\Psi_{12, i}^{n}, 1 \leq i \leq n$, until the initial configuration is attained.

The extension of the symmetry, outlined in Table 1, to the special case, $n=12$, implies Euclidean 0 -spaces, $\Re^{0}$, to be taken into consideration. Accordingly, $\Psi_{12}^{0}$ would lie outside $\Re^{0}$, on a coordinate axis at a distance, $x_{0}=12$, from the origin which, in turn, coincides with $\Re^{0}$. Then the boundary condition, expressed by Eq. (1), is satisfied.

At this stage, a nontrivial question is if $n$-chords of each cardinality can be enumerated within the framework of the geometrical interpretation outlined above. Let $n$-chords exhibiting distinct coordinates be first considered.

For $n=0$, the Euclidean 0 -space, $\Re^{0}$, reduces to the origin and Eq. (11) still holds but with regard to a point of coordinate, $\ell_{0}=12$, outside $\Re^{0}$. Accordingly, $N_{\mathrm{C}}(0)=0$ as listed in Table 1.

For $n=1$, the Euclidean 1 -space, $\Re^{1}$, reduces to the real axis, where Eq. (11) is satisfied on the point of coordinate, $\ell_{1}=12$. Accordingly, $N_{\mathrm{C}}(1)=$ 1 , as listed in Table 1,

For $n=2$, the Euclidean 2-space, $\Re^{2}$, reduces to a plane, where Eq. (1) is satisfied on the points of coordinates, $\left(\ell_{1}, 12-\ell_{1}\right), 1 \leq \ell_{1} \leq 11$. The above mentioned points are displaced on a regular inclined 2-hedron (regular side), $\psi_{12}^{2}$, of vertexes, $\mathrm{V}_{\mathrm{i}}^{\prime} \equiv\left(1+10 \delta_{1 i}, 1+10 \delta_{2 i}\right), 1 \leq i \leq 2$, in number of eleven. Accordingly, the number of distinct 2-chords must be counted along a series of superimposed 1-hedrons (regular vertexes) through the second dimension. The result is $N_{\mathrm{C}}(2)=11$, as listed in Table 1 .

For $n=3$, the Euclidean 3 -space, $\Re^{3}$, is an ordinary space, where Eq. (11) is satisfied on the points of coordinates, $\left(\ell_{1}, \ell_{2}, 12-\ell_{1}-\ell_{2}\right), 1 \leq \ell_{k} \leq 10$, $k=1,2$. As shown in Fig. 3, the above mentioned points are displaced on a regular inclined 3-hedron (regular triangle), $\psi_{12}^{3}$, of vertexes, $\mathrm{V}_{\mathrm{i}}^{\prime} \equiv\left(1+9 \delta_{1 i}, 1+\right.$ $\left.9 \delta_{2 i}, 1+9 \delta_{3 i}\right), 1 \leq i \leq 3$, in number of ten on each 1 -face (regular side) and scaled by one passing to the next related 1-hedron up to the opposite vertex. 
Accordingly, the number of distinct 3-chords must be counted along a series of superimposed 2-hedrons through the third dimension. The result is:

$$
N_{\mathrm{C}}(3)=\sum_{k=1}^{10} k=10+9+8+7+6+5+4+3+2+1=\frac{11 \cdot 10}{2}=55 ;
$$

as listed in Table 1 .

For $n=4$, Eq. (11) is satisfied in $\Re^{4}$ on the points of coordinates, $\left(\ell_{1}, \ell_{2}, \ell_{3}\right.$, $\left.12-\ell_{1}-\ell_{2}-\ell_{3}\right), 1 \leq \ell_{k} \leq 9, k=1,2,3$. The above mentioned points are displaced on a regular inclined 4-hedron (regular tetrahedron), $\psi_{12}^{4}$, of vertexes, $\bigvee_{i}^{\prime} \equiv\left(1+8 \delta_{1 i}, 1+8 \delta_{2 i}, 1+8 \delta_{3 i}, 1+8 \delta_{4 i}\right), 1 \leq i \leq 4$, in number of nine on each 1 -face, yielding $10 \cdot 9 / 2=45$ points on each 2 -face. Accordingly, the number of distinct 4-chords must be counted along a series of superimposed 3-hedrons through the fourth dimension. The result is:

$$
\begin{aligned}
N_{\mathrm{C}}(4) & =\sum_{k=1}^{9} k+\sum_{k=1}^{8} k+\sum_{k=1}^{7} k+\sum_{k=1}^{6} k+\sum_{k=1}^{5} k+\sum_{k=1}^{4} k+\sum_{k=1}^{3} k+\sum_{k=1}^{2} k+\sum_{k=1}^{1} k \\
& =5 \cdot 9+4 \cdot 9+4 \cdot 7+3 \cdot 7+3 \cdot 5+2 \cdot 5+2 \cdot 3+1 \cdot 3+1 \cdot 1 \\
& =9^{2}+7^{2}+5^{2}+3^{2}+1^{2}=165
\end{aligned}
$$

as listed in Table 1

For generic $n$, Eq. (1) is satisfied in $\Re^{n}$ on the points of coordinates, $\left(\ell_{1}, \ldots, \ell_{n-1}, 12-\ell_{1}-\ldots-\ell_{n-1}\right), 1 \leq \ell_{k} \leq 12-n+1, k=1,2, \ldots, n-1$. The above mentioned points are displaced on a regular inclined $n$-hedron, $\psi_{12}^{n}$, of vertexes, $\mathrm{V}_{\mathrm{i}}^{\prime} \equiv\left[1+(12-n) \delta_{1 i}, 1+(12-n) \delta_{2 i}, \ldots, 1+(12-n) \delta_{n i}\right], 1 \leq i \leq n$, in number of $12-n+1$ on each 1 -face, yielding $(12-n+2)(12-n+1) / 2$ points on each 2 -face. Accordingly, the number of distinct $n$-chords must be counted along a series of superimposed $(n-1)$-hedrons through the $n$th dimension. The result is:

$$
N_{\mathrm{C}}(n)=N_{\mathrm{C}}\left(\tau_{12-n+1}^{(n-2)}\right)+N_{\mathrm{C}}\left(\tau_{12-n}^{(n-2)}\right)+\ldots+N_{\mathrm{C}}\left(\tau_{1}^{(n-2)}\right) \quad ; \quad n \geq 2 ;
$$

where $N_{\mathrm{C}}\left(\tau_{k}^{(n-2)}\right)$ represents the number of distinct $n$-chords within the $(n-1)$-hedron of the series, $\tau_{k}^{(n-2)}, 1 \leq k \leq 12-n+1$, including on each 1 -face $k$ positive integer points which satisfy Eq. (1). More specifically, the generic term on the right-hand side of Eq. (48) can be expressed as:

$$
N_{\mathrm{C}}\left(\tau_{k}^{(n-2)}\right)=N_{\mathrm{C}}\left(\tau_{k+1}^{(n-2)}\right)-N_{\mathrm{C}}\left(\tau_{k+1}^{(n-3)}\right) \quad ; \quad n \geq 2 ;
$$

where $N_{\mathrm{C}}\left(\tau_{k+1}^{(n-3)}\right)$ is the counterpart of $N_{\mathrm{C}}\left(\tau_{k+1}^{(n-2)}\right)$ with regard to the $(n-2)$ hedron of the related series in $\Re^{n-1}$, and $N_{\mathrm{C}}\left(\tau_{12-n+2}^{(n-2)}\right)=N_{\mathrm{C}}(n-1)$. Then 
each term on the right-hand side of Eq. (48) can be determined via Eq. (49), provided its counterpart in $\Re^{n-1}$ is known.

The particularization of Eq. (49) to $n=5$ via (47) yields:

$$
\begin{aligned}
& N_{\mathrm{C}}\left(\tau_{8}^{(3)}\right)=N_{\mathrm{C}}(4)-N_{\mathrm{C}}\left(\tau_{9}^{(2)}\right)=165-45=120 ; \\
& N_{\mathrm{C}}\left(\tau_{7}^{(3)}\right)=N_{\mathrm{C}}\left(\tau_{8}^{(3)}\right)-N_{\mathrm{C}}\left(\tau_{8}^{(2)}\right)=120-36=84 ; \\
& N_{\mathrm{C}}\left(\tau_{6}^{(3)}\right)=N_{\mathrm{C}}\left(\tau_{7}^{(3)}\right)-N_{\mathrm{C}}\left(\tau_{7}^{(2)}\right)=84-28=56 ; \\
& N_{\mathrm{C}}\left(\tau_{5}^{(3)}\right)=N_{\mathrm{C}}\left(\tau_{6}^{(3)}\right)-N_{\mathrm{C}}\left(\tau_{6}^{(2)}\right)=56-21=35 ; \\
& N_{\mathrm{C}}\left(\tau_{4}^{(3)}\right)=N_{\mathrm{C}}\left(\tau_{5}^{(3)}\right)-N_{\mathrm{C}}\left(\tau_{5}^{(2)}\right)=35-15=20 ; \\
& N_{\mathrm{C}}\left(\tau_{3}^{(3)}\right)=N_{\mathrm{C}}\left(\tau_{4}^{(3)}\right)-N_{\mathrm{C}}\left(\tau_{4}^{(2)}\right)=20-10=10 ; \\
& N_{\mathrm{C}}\left(\tau_{2}^{(3)}\right)=N_{\mathrm{C}}\left(\tau_{3}^{(3)}\right)-N_{\mathrm{C}}\left(\tau_{3}^{(2)}\right)=10-6=4 ; \\
& N_{\mathrm{C}}\left(\tau_{1}^{(3)}\right)=N_{\mathrm{C}}\left(\tau_{2}^{(3)}\right)-N_{\mathrm{C}}\left(\tau_{2}^{(2)}\right)=4-3=1 ;
\end{aligned}
$$

and the particularization of Eq. (48) to $n=5$ via (50) yields the number of distinct 5-chords as:

$$
N_{\mathrm{C}}(5)=120+84+56+35+20+10+4+1=330 ;
$$

in accordance with Table 1.

The particularization of Eq. (49) to $n=6$ via (50)-(51) yields:

$$
\begin{aligned}
& N_{\mathrm{C}}\left(\tau_{7}^{(4)}\right)=N_{\mathrm{C}}(5)-N_{\mathrm{C}}\left(\tau_{8}^{(3)}\right)=330-120=210 ; \\
& N_{\mathrm{C}}\left(\tau_{6}^{(4)}\right)=N_{\mathrm{C}}\left(\tau_{7}^{(4)}\right)-N_{\mathrm{C}}\left(\tau_{7}^{(3)}\right)=210-84=126 ; \\
& N_{\mathrm{C}}\left(\tau_{5}^{(4)}\right)=N_{\mathrm{C}}\left(\tau_{6}^{(4)}\right)-N_{\mathrm{C}}\left(\tau_{6}^{(3)}\right)=126-56=70 ; \\
& N_{\mathrm{C}}\left(\tau_{4}^{(4)}\right)=N_{\mathrm{C}}\left(\tau_{5}^{(4)}\right)-N_{\mathrm{C}}\left(\tau_{5}^{(3)}\right)=70-35=35 ; \\
& N_{\mathrm{C}}\left(\tau_{3}^{(4)}\right)=N_{\mathrm{C}}\left(\tau_{4}^{(4)}\right)-N_{\mathrm{C}}\left(\tau_{4}^{(3)}\right)=35-20=15 ; \\
& N_{\mathrm{C}}\left(\tau_{2}^{(4)}\right)=N_{\mathrm{C}}\left(\tau_{3}^{(4)}\right)-N_{\mathrm{C}}\left(\tau_{3}^{(3)}\right)=15-10=5 ; \\
& N_{\mathrm{C}}\left(\tau_{1}^{(4)}\right)=N_{\mathrm{C}}\left(\tau_{2}^{(4)}\right)-N_{\mathrm{C}}\left(\tau_{2}^{(3)}\right)=5-4=1 ;
\end{aligned}
$$

and the particularization of Eq. (48) to $n=6$ via (52) yields the number of distinct 6-chords as:

$$
N_{\mathrm{C}}(6)=210+126+70+35+15+5+1=462 ;
$$

in accordance with Table 1. 
The particularization of Eq. (49) to $n=7$ via (52)-(53) yields:

$$
\begin{aligned}
& N_{\mathrm{C}}\left(\tau_{6}^{(5)}\right)=N_{\mathrm{C}}(6)-N_{\mathrm{C}}\left(\tau_{7}^{(4)}\right)=462-210=252 ; \\
& N_{\mathrm{C}}\left(\tau_{5}^{(5)}\right)=N_{\mathrm{C}}\left(\tau_{6}^{(5)}\right)-N_{\mathrm{C}}\left(\tau_{6}^{(4)}\right)=252-126=126 ; \\
& N_{\mathrm{C}}\left(\tau_{4}^{(5)}\right)=N_{\mathrm{C}}\left(\tau_{5}^{(5)}\right)-N_{\mathrm{C}}\left(\tau_{5}^{(4)}\right)=126-70=56 ; \\
& N_{\mathrm{C}}\left(\tau_{3}^{(5)}\right)=N_{\mathrm{C}}\left(\tau_{4}^{(5)}\right)-N_{\mathrm{C}}\left(\tau_{4}^{(4)}\right)=56-35=21 ; \\
& N_{\mathrm{C}}\left(\tau_{2}^{(5)}\right)=N_{\mathrm{C}}\left(\tau_{3}^{(5)}\right)-N_{\mathrm{C}}\left(\tau_{3}^{(4)}\right)=21-15=6 ; \\
& N_{\mathrm{C}}\left(\tau_{1}^{(5)}\right)=N_{\mathrm{C}}\left(\tau_{2}^{(5)}\right)-N_{\mathrm{C}}\left(\tau_{2}^{(4)}\right)=6-5=1 ;
\end{aligned}
$$

and the particularization of Eq. (48) to $n=7$ via (54) yields the number of distinct 7-chords as:

$$
N_{\mathrm{C}}(7)=252+126+56+21+6+1=462 ;
$$

in accordance with Table 1.

The particularization of Eq. (49) to $n=8$ via (54)-(55) yields:

$$
\begin{aligned}
& N_{\mathrm{C}}\left(\tau_{5}^{(6)}\right)=N_{\mathrm{C}}(7)-N_{\mathrm{C}}\left(\tau_{6}^{(5)}\right)=462-252=210 ; \\
& N_{\mathrm{C}}\left(\tau_{4}^{(6)}\right)=N_{\mathrm{C}}\left(\tau_{5}^{(6)}\right)-N_{\mathrm{C}}\left(\tau_{5}^{(5)}\right)=210-126=84 ; \\
& N_{\mathrm{C}}\left(\tau_{3}^{(6)}\right)=N_{\mathrm{C}}\left(\tau_{4}^{(6)}\right)-N_{\mathrm{C}}\left(\tau_{4}^{(5)}\right)=84-56=28 ; \\
& N_{\mathrm{C}}\left(\tau_{2}^{(6)}\right)=N_{\mathrm{C}}\left(\tau_{3}^{(6)}\right)-N_{\mathrm{C}}\left(\tau_{3}^{(5)}\right)=28-21=7 ; \\
& N_{\mathrm{C}}\left(\tau_{1}^{(6)}\right)=N_{\mathrm{C}}\left(\tau_{2}^{(6)}\right)-N_{\mathrm{C}}\left(\tau_{2}^{(5)}\right)=7-6=1 ;
\end{aligned}
$$

and the particularization of Eq. (48) to $n=8$ via (56) yields the number of distinct 8-chords as:

$$
N_{\mathrm{C}}(8)=210+84+28+7+1=330 ;
$$

in accordance with Table 1.

The particularization of Eq. (49) to $n=9$ via (56)-(57) yields:

$$
\begin{aligned}
& N_{\mathrm{C}}\left(\tau_{4}^{(7)}\right)=N_{\mathrm{C}}(8)-N_{\mathrm{C}}\left(\tau_{5}^{(6)}\right)=330-210=120 ; \\
& N_{\mathrm{C}}\left(\tau_{3}^{(7)}\right)=N_{\mathrm{C}}\left(\tau_{4}^{(7)}\right)-N_{\mathrm{C}}\left(\tau_{4}^{(6)}\right)=120-84=36 ; \\
& N_{\mathrm{C}}\left(\tau_{2}^{(7)}\right)=N_{\mathrm{C}}\left(\tau_{3}^{(7)}\right)-N_{\mathrm{C}}\left(\tau_{3}^{(6)}\right)=36-28=8 ; \\
& N_{\mathrm{C}}\left(\tau_{1}^{(7)}\right)=N_{\mathrm{C}}\left(\tau_{2}^{(7)}\right)-N_{\mathrm{C}}\left(\tau_{2}^{(6)}\right)=8-7=1 ;
\end{aligned}
$$


and the particularization of Eq. (48) to $n=9$ via (58) yields the number of distinct 9-chords as:

$$
N_{\mathrm{C}}(9)=120+36+8+1=165 ;
$$

in accordance with Table 1.

The particularization of Eq. (49) to $n=10$ via (58)-(59) yields:

$$
\begin{aligned}
& N_{\mathrm{C}}\left(\tau_{3}^{(8)}\right)=N_{\mathrm{C}}(9)-N_{\mathrm{C}}\left(\tau_{4}^{(7)}\right)=165-120=45 ; \\
& N_{\mathrm{C}}\left(\tau_{2}^{(8)}\right)=N_{\mathrm{C}}\left(\tau_{3}^{(8)}\right)-N_{\mathrm{C}}\left(\tau_{3}^{(7)}\right)=45-36=9 ; \\
& N_{\mathrm{C}}\left(\tau_{1}^{(8)}\right)=N_{\mathrm{C}}\left(\tau_{2}^{(8)}\right)-N_{\mathrm{C}}\left(\tau_{2}^{(7)}\right)=9-8=1 ;
\end{aligned}
$$

and the particularization of Eq. (48) to $n=10$ via (60) yields the number of distinct 10-chords as:

$$
N_{\mathrm{C}}(10)=45+9+1=55 ;
$$

in accordance with Table 1.

The particularization of Eq. (49) to $n=11$ via (60)-(61) yields:

$$
\begin{aligned}
& N_{\mathrm{C}}\left(\tau_{2}^{(9)}\right)=N_{\mathrm{C}}(10)-N_{\mathrm{C}}\left(\tau_{3}^{(8)}\right)=55-45=10 \\
& N_{\mathrm{C}}\left(\tau_{1}^{(9)}\right)=N_{\mathrm{C}}\left(\tau_{2}^{(9)}\right)-N_{\mathrm{C}}\left(\tau_{2}^{(8)}\right)=10-9=1 ;
\end{aligned}
$$

and the particularization of Eq. (48) to $n=11$ via (62) yields the number of distinct 11-chords as:

$$
N_{\mathrm{C}}(11)=10+1=11 ;
$$

in accordance with Table 1 .

The particularization of Eq. (49) to $n=12$ via (62)-(63) yields:

$$
N_{\mathrm{C}}\left(\tau_{1}^{(10)}\right)=N_{\mathrm{C}}(11)-N_{\mathrm{C}}\left(\tau_{2}^{(9)}\right)=11-10=1
$$

and the particularization of Eq. (48) to $n=12$ via (64) yields the number of distinct 12-chords as:

$$
N_{\mathrm{C}}(12)=1
$$

in accordance with Table 1.

In summary, Eqs. (46)-(65) disclose Eq. (15) can be inferred via geometric as well as algebraic considerations, but the former alternative deserves further attention. To this respect, let regular, inclined $n$-hedrons, $\Psi_{12}^{n}$, of vertexes, 
$\mathrm{V}_{\mathrm{i}} \equiv\left(12 \delta_{1 i}, 12 \delta_{2 i}, \ldots, 12 \delta_{n i}\right), 1 \leq i \leq n$, be considered as special cases of lattice polytopes in $\Re^{n}$ e.g., [22].

In this view, Eq. (5) yields the number of lattice points (i.e. points of integer coordinates) within $\Psi_{12}^{n}$, while the total number reads:

$$
N_{\mathrm{V}}(n)=N_{\mathrm{C}}(n)+N_{\mathrm{S}}(n) ;
$$

where $N_{\mathrm{S}}(n)$ is the number of lattice points on the $(n-2)$-surface of $\Psi_{12}^{n}$, which necessarily exhibit at least one null coordinate. In the following, $N_{\mathrm{S}}(n)$ and $N_{\mathrm{V}}(n)$ shall be determined for each cardinality, keeping in mind values of $N_{\mathrm{C}}(n)$ are listed in Table 1 .

For $n=0$, no lattice polytope exists in the sense $\Psi_{12}^{0}$ lies outside $\Re^{0}$. Accordingly, $N_{\mathrm{S}}(0)=0, N_{\mathrm{V}}(0)=0$.

For $n=1, \Psi_{12}^{1}$ reduces to a regular vertex, $\mathrm{V}_{\mathrm{i}} \equiv\left(12 \delta_{1 i}\right)$. Accordingly, $N_{\mathrm{S}}(1)=0, N_{\mathrm{V}}(1)=N_{\mathrm{C}}(1)=1$.

For $n=2, \Psi_{12}^{2}$ reduces to a regular side of extremes, $\mathrm{V}_{\mathbf{i}} \equiv\left(12 \delta_{1 i}, 12 \delta_{2 i}\right)$. Accordingly, $N_{\mathrm{S}}(2)=1 \cdot 2=2, N_{\mathrm{V}}(2)=11+2=13$.

For $n=3, \Psi_{12}^{3}$ reduces to a regular triangle of vertexes, $\mathrm{V}_{\mathrm{i}} \equiv\left(12 \delta_{1 i}, 12 \delta_{2 i}, 12 \delta_{3 i}\right)$, as shown in Fig.2. Lattice points exhibiting at least one null coordinate lie on the principal planes, in number of 12 provided vertexes (exhibiting two null coordinates) are partitioned one per principal plane. Accordingly, $N_{\mathrm{S}}(2)=3 \cdot 12=36, N_{\mathrm{V}}(2)=55+36=91$.

In general, passing from $\Re^{n-1}$ to $\Re^{n}$, the number of lattice points within $\Psi_{12}^{n-1}$ has to be incremented by the number of lattice points within $\Psi_{12}^{n-2}$ for including points exhibiting at least one null coordinate, which makes the number of lattice points on a $(n-2)$-face of $\Psi_{12}^{n}$ i.e. $1 / n$ the number of lattice points on the $(n-2)$-surface of $\Psi_{12}^{n}$. Accordingly, the number of lattice points on the $(n-2)$-surface of $\Psi_{12}^{n}$ reads:

$$
N_{\mathrm{S}}(n)=n\left[N_{\mathrm{C}}(n-1)+N_{\mathrm{C}}(n-2)\right] ; \quad n>1 ;
$$

which, for $n=2,3$, reproduces the above results.

Using Eqs. (66)-(67) for each cardinality yields:

$$
\begin{aligned}
& N_{\mathrm{S}}(2)=2(1+0)=2 ; \quad N_{\mathrm{V}}(2)=11+2=13 ; \\
& N_{\mathrm{S}}(3)=3(11+1)=36 ; \quad N_{\mathrm{V}}(3)=55+36=91 ; \\
& N_{\mathrm{S}}(4)=4(55+11)=264 ; \quad N_{\mathrm{V}}(4)=165+264=429 ; \\
& N_{\mathrm{S}}(5)=5(165+55)=1100 ; \quad N_{\mathrm{V}}(5)=330+1100=1430 ; \\
& N_{\mathrm{S}}(6)=6(330+165)=2970 ; \quad N_{\mathrm{V}}(6)=462+2970=3432 ; \\
& N_{\mathrm{S}}(7)=7(462+330)=5544 ; \quad N_{\mathrm{V}}(7)=462+5544=6006 \quad ; \\
& N_{\mathrm{S}}(8)=8(462+462)=7392 ; \quad N_{\mathrm{V}}(8)=330+7392=7722 ;
\end{aligned}
$$


Table 5: Number of lattice points within the $(n-1)$-volume, $N_{\mathrm{C}}$, and on the $(n-2)$-surface, $N_{\mathrm{S}}$, of $\Psi_{12}^{n}$ polytopes, and total amount, $N_{\mathrm{V}}=N_{\mathrm{C}}+N_{\mathrm{S}}$. See text for further details.

\begin{tabular}{|c|r|r|r|r|r|r|r|r|r|r|r|r|r|}
\hline \hline$n$ & 0 & 1 & 2 & 3 & 4 & 5 & 6 & 7 & 8 & 9 & 10 & 11 & 12 \\
$N_{\mathrm{C}}$ & 0 & 1 & 11 & 55 & 165 & 330 & 462 & 462 & 330 & 165 & 55 & 11 & 1 \\
$N_{\mathrm{S}}$ & 0 & 0 & 2 & 36 & 264 & 1110 & 2970 & 5944 & 7392 & 7128 & 4950 & 2420 & 792 \\
$N_{\mathrm{V}}$ & 0 & 1 & 13 & 91 & 429 & 1430 & 3432 & 6006 & 7722 & 7293 & 5005 & 2431 & 793 \\
\hline
\end{tabular}

$$
\begin{aligned}
& N_{\mathrm{S}}(9)=9(330+462)=7128 ; \quad N_{\mathrm{V}}(9)=165+7128=7293 ; \\
& N_{\mathrm{S}}(10)=10(165+330)=4950 ; \quad N_{\mathrm{V}}(10)=55+4950=5005 \\
& N_{\mathrm{S}}(11)=11(55+165)=2420 ; \quad N_{\mathrm{V}}(11)=11+2420=2431 ; \\
& N_{\mathrm{S}}(12)=12(11+55)=792 ; \quad N_{\mathrm{V}}(12)=1+792=793 ;
\end{aligned}
$$

which are listed in Table 5 together with $N_{\mathrm{C}}(n)$, the last repeated for better comparison.

At this stage, what remains to be done is the enumeration of repeating $n$-chords within the framework of the current geometrical interpretation. To this respect, it is worth remembering $\Psi_{12}^{n}$ can be partitioned into $n$ congruent $\Psi_{12, i}^{n}, 1 \leq i \leq n$, where different $n$-chords within an assigned $T_{n}$ or $T_{n} / T_{n} I$ set class relate to points which are similarly placed within different $\Psi_{12, i}^{n}$. In this view, repeating $n$-chords necessarily lie on the boundary between different $\Psi_{12, i}^{n}$ i.e. $(n-2)$-faces, and must be counted twice, three times, or more, according if points under consideration are common to two, three, or more $\Psi_{12, i}^{n}$. Then repeating $n$-chords are expected to relate to some kind of symmetric points, lying on the straight lines joining the orthocentres of $(j-1)$-faces, $1 \leq j \leq n-2$.

For $n=0, \Psi_{12}^{0}$ lies outside $\Re^{0}$, which implies no repeating 0 -chords.

For $n=1, \Psi_{12}^{1}=\Psi_{12,1}^{1}$, which implies no repeating 1-chords.

For $n=2, \Psi_{12,1}^{2}$ and $\Psi_{12,2}^{2}$ have in common the orthocentre of $\Psi_{12}^{2}, \mathrm{H}_{2} \equiv$ $(6,6)$, which has to be counted twice for being equally partitioned among $\Psi_{12,1}^{2}$ and $\Psi_{12,2}^{2}$, for a total of repeating 1 . In summary, the number of repeating 2-chords is 1 , as shown in Table 2 .

For $n=3, \Psi_{12,1}^{3}, \Psi_{12,2}^{3}, \Psi_{12,3}^{3}$, have in common the orthocentre of $\Psi_{12}^{3}$, $\mathrm{H}_{3} \equiv(4,4,4)$, which has to be counted three times for being equally partitioned among $\Psi_{12,1}^{3}, \Psi_{12,2}^{3}, \Psi_{12,3}^{3}$, for a total of repeating 2 . In summary, the number of repeating 3 -chords is 2 , as shown in Table 2 .

For $n=4, \Psi_{12, i}^{4}, 1 \leq i \leq 4$, have in common the orthocentre of $\Psi_{12}^{4}, \mathrm{H}_{4} \equiv$ 
$(3,3,3,3)$, which has to be counted four times for being equally partitioned among $\Psi_{12, i}^{4}, 1 \leq i \leq 4$, for a total of repeating 3 . In addition, pairs of 2 -faces (regular triangles) have in common orthocentres of 1-faces (regular sides) on the boundary, $\mathrm{H}_{4,2} \equiv\left[\ell+k\left(\delta_{1 i}+\delta_{1 j}\right), \ell+k\left(\delta_{2 i}+\delta_{2 j}\right), \ldots, \ell+k\left(\delta_{4 i}+\delta_{4 j}\right)\right], \ell=1,2$, $k=4,2$. The number of 1 -face orthocentres equals 6 in any case, where 4 are equally partitioned among $\Psi_{12, i}^{4}$, while the remaining $2,\{x, y, x, y\}$, $\{y, x, y, x\}$, must necessarily be counted twice for a total of repeating $2+2=4$. In summary, the number of repeating 4 -chords is $3+4=7$, as shown in Table 2 .

For $n=5$, the orthocentre of $\Psi_{12}^{5}$, is not an integer point. On the other hand, $(j-1)$-faces of $\Psi_{12}^{5}, 0<j<4$, occur in multiples of 5 as shown in Table 7, which implies related orthocentres are equally partitioned among $\Psi_{12, i}^{5}, 1 \leq i \leq 5$, and repeating 5 -chords do not take place, according to Table 2.

For $n=6, \Psi_{12, i}^{6}, 1 \leq i \leq 6$, have in common the orthocentre of $\Psi_{12}^{6}$, $\mathrm{H}_{6} \equiv(2,2,2,2,2,2)$, which has to be counted six times for being equally partitioned among $\Psi_{12, i}^{6}, 1 \leq i \leq 6$, as shown in Table 2. In addition, pairs of 2-faces (regular triangles) have in common orthocentres of 1-faces (regular sides) on the boundary, $\mathrm{H}_{6,2} \equiv\left[\ell+k\left(\delta_{1 i}+\delta_{1 j}\right), \ell+k\left(\delta_{2 i}+\delta_{2 j}\right), \ldots, \ell+k\left(\delta_{6 i}+\delta_{6 j}\right)\right]$, $\ell=1, k=3$. The number of 1 -face orthocentres equals 15 , where 12 are equally partitioned among $\Psi_{12, i}^{6}$, while the remaining $3,\{x, x, y, x, x, y\}$, $\{x, y, x, x, y, x\},\{y, x, x, y, x, x\}$, must necessarily be counted twice for a total of repeating $1+1+1=3$. Furthermore, pairs of 3 -faces (regular tetrahedrons) have in common orthocentres of 2-faces (regular triangles) on the boundary, $\mathrm{H}_{6,3} \equiv\left[\ell+k\left(\delta_{1 i}+\delta_{1 j}+\delta_{1 \ell}\right), \ell+k\left(\delta_{2 i}+\delta_{2 j}+\delta_{2 \ell}\right), \ldots, \ell+k\left(\delta_{6 i}+\delta_{6 j}+\delta_{6 \ell}\right)\right], \ell=1$, $k=2$. The number of 2 -face orthocentres equals 20 , where 18 are equally partitioned among $\Psi_{12, i}^{6}$, while the remaining $2,\{x, y, x, y, x, y\},\{y, x, y, x, y, x\}$, must necessarily be counted three times for a total of repeating $2+2=4$. Finally, triads of 3 -faces (regular tetrahedrons) have in common symmetric points with respect to the above mentioned orthocentres, exhibiting identical pairs of coordinates e.g., $\{x, x, y, y, z, z\}, z=(x+y) / 2$. The total number of pairs within a 6 -tuple is $\left(\begin{array}{l}6 \\ 2\end{array}\right)=15$, and the total number of pairs within the remaining 4 -tuple is $\left(\begin{array}{l}4 \\ 2\end{array}\right)=6$, while the remaining 2 -tuple is fixed. Accordingly, the total number of 6 -tuples with pairs of identical coordinates equals $15 \cdot 6=90$, where 84 are equally partitioned among $\Psi_{12, i}^{6}$, while the remaining $6,\{x, y, z, x, y, z\},\{y, z, x, y, z, x\},\{z, x, y, z, x, y\} ;\{x, z, y, x, z, y\}$, $\{z, y, x, z, y, x\},\{y, x, z, y, x, z\}$; must necessarily be counted twice for a total of repeating $(1+1+1)+(1+1+1)=3+3=6$. In summary, the number of repeating $n$-chords is $5+3+4+6=18$, as shown in Table 2 .

For $n=7$, the orthocentre of $\Psi_{12}^{7}$, is not an integer point. On the other 
hand, $(j-1)$-faces of $\Psi_{12}^{7}, 0<j<6$, occur in multiples of 7 as shown in Table 7, which implies related orthocentres are equally partitioned among $\Psi_{12, i}^{7}, 1 \leq i \leq 7$, and repeating 7 -chords do not take place, according to Table 2 .

For $n=8$, the orthocentre of $\Psi_{12}^{8}$, is not an integer point. On the other hand, pairs of 2-faces (regular triangles) have in common orthocentres of 1 -faces (regular sides) on the boundary, $\mathrm{H}_{8,2} \equiv\left[\ell+k\left(\delta_{1 i}+\delta_{1 j}\right), \ell+\right.$ $\left.k\left(\delta_{2 i}+\delta_{2 j}\right), \ldots, \ell+k\left(\delta_{8 i}+\delta_{8 j}\right)\right], \ell=1, k=2$. The number of 1-face orthocentres equals 28 , where 24 are equally partitioned among $\Psi_{12, i}^{8}$, while the remaining $4,\{x, x, x, y, x, x, x, y\},\{x, x, y, x, x, x, y, x\},\{x, y, x, x, x, y, x, x\}$, $\{y, x, x, x, y, x, x, x\}$, must necessarily be counted twice for a total of repeating $1+1+1+1=4$. In addition, pairs of 4 -faces have in common orthocentres of 3 -faces (regular tetrahedrons) on the boundary, $\mathrm{H}_{8,4} \equiv\left[\ell+k\left(\delta_{1 i_{1}}+\delta_{1 i_{2}}+\right.\right.$ $\left.\left.\delta_{1 i_{3}}+\delta_{1 i_{4}}\right), \ell+k\left(\delta_{2 i_{1}}+\delta_{2 i_{2}}+\delta_{2 i_{3}}+\delta_{2 i_{4}}\right), \ldots, \ell+k\left(\delta_{8 i_{1}}+\delta_{8 i_{2}}+\delta_{8 i_{3}}+\delta_{8 i_{4}}\right)\right]$, $\ell=1, k=2$. The number of 3 -face orthocentres equals 70 , where 64 are equally partitioned among $\Psi_{12, i}^{8}$, while the remaining $6,\{x, y, x, y, x, y, x, y\}$, $\{y, x, y, x, y, x, y, x\} ;\{x, x, y, y, x, x, y, y\},\{x, y, y, x, x, y, y, x\},\{y, y, x, x, y, y, x, x\}$, $\{y, x, x, y, y, x, x, y\}$; must necessarily be counted four times in the former case and twice in the latter, for a total of repeating $3+3=6$ and $1+1+1+1=4$, respectively, yielding $6+4=10$. In summary, the number of repeating 8 -chords is $4+10=14$, as shown in Table 2 ,

For $n=9$, the orthocentre of $\Psi_{12}^{9}$, is not an integer point. On the other hand, pairs of 3-faces (regular tetrahedrons) have in common orthocentres of 2 -faces (regular triangles) on the boundary, $\mathrm{H}_{9,3} \equiv\left[\ell+k\left(\delta_{1 i}+\delta_{1 j}+\delta_{1 m}\right), \ell+\right.$ $\left.k\left(\delta_{2 i}+\delta_{2 j}+\delta_{2 m}\right), \ldots, \ell+k\left(\delta_{9 i}+\delta_{9 j}+\delta_{9 m}\right)\right], \ell=1, k=2$. The number of 2 -face orthocentres equals 84 , where 81 are equally partitioned among $\Psi_{12, i}^{9}$, while the remaining $3,\{x, x, y, x, x, y, x, x, y\},\{x, y, x, x, y, x, x, y, x\}$, $\{y, x, x, y, x, x, y, x, x\}$, must necessarily be counted three times for a total of repeating $2+2+2=6$. In summary, the number of repeating 9 -chords equals 6 , as shown in Table 2 .

For $n=10$, the orthocentre of $\Psi_{12}^{10}$, is not an integer point. On the other hand, pairs of 2-faces (regular triangles) have in common orthocentres of 1faces (regular sides) on the boundary, $\mathrm{H}_{10,2} \equiv\left[\ell+k\left(\delta_{1 i}+\delta_{1 j}\right), \ell+k\left(\delta_{2 i}+\right.\right.$ $\left.\left.\delta_{2 j}\right), \ldots, \ell+k\left(\delta_{10, i}+\delta_{10, j}\right)\right], \ell=1, k=1$. The number of 1 -face orthocentres equals 45 , where 40 are equally partitioned among $\Psi_{12, i}^{10}$, while the remaining $5,\{x, x, x, x, y, x, x, x, x, y\},\{x, x, x, y, x, x, x, x, y, x\},\{x, x, y, x, x, x, x, y, x, x\}$, $\{x, y, x, x, x, x, y, x, x, x\},\{y, x, x, x, x, y, x, x, x, x\}$, must necessarily be counted twice for a total of repeating $1+1+1+1+1=5$. In summary, the number of repeating 10-chords equals 5 , as shown in Table 2 .

For $n=11$, the orthocentre of $\Psi_{12}^{11}$, is not an integer point. On the other hand, $(j-1)$-faces of $\Psi_{12}^{11}, 0<j<10$, occur in multiples of 11 as shown 
in Table 7, which implies related orthocentres are equally partitioned among $\Psi_{12, i}^{11}, 1 \leq i \leq 11$, and repeating 11 -chords do not take place, according to Table 2 .

For $n=12, \Psi_{12, i}^{12}, 1 \leq i \leq 12$, have in common the orthocentre of $\Psi_{12}^{12}$, $\mathrm{H}_{12} \equiv(1,1, \ldots, 1)$, which has to be counted twelve times for being equally partitioned among $\Psi_{12, i}^{12}, 1 \leq i \leq 12$. Keeping in mind no additional positive integer point belongs to $\Psi_{12}^{12}$, the number of repeating 12-chords equals 11 , as shown in Table 2.

The above results disclose both distinct and repeating $n$-chords and $T_{n}$ or $T_{n} / T_{n} I$ set classes may be enumerated in the light of a geometrical interpretation, reproducing results inferred via an algebraic approach. To this respect, it is worth noticing pairwise $T_{n}$ and $T_{n} I$ set classes, made of repeating but neither palindrome nor pseudo palindrome $n$-chords, are different, which implies a $2: 1$ correspondence between $T_{n}$ and $T_{n} / T_{n} I$ set classes. Conversely, pairwise $T_{n}$ and $T_{n} I$ set classes, made of repeating palindrome or pseudo palindrome $n$-chords, are coinciding, which implies a $1: 1$ correspondence between $T_{n}$ and $T_{n} / T_{n} I$ set classes.

For further details on $\Psi_{12}^{n} n$-hedrons, an interested reader is addressed to Appendix C.

\section{Discussion}

Concerning musical intervals in multiples of semitones under 12-note equal temperament, counting pitch-class sets with respect to an assigned equivalence relation lies between two extreme cases, namely brute-force listing and elegant (but esoteric) enumeration procedure from group theory. The method exploited in Section 2 implies, on one hand, determination of repeating and palindrome or pseudo palindrome $n$-chords by use, on the other hand, of a less elegant (but less esoteric) enumeration procedure. More specifically, $n$-chords of cardinality, $n$, are related to positive integer points in $\Re^{n}$, belonging to a regular inclined $n$-hedron, $\Psi_{12}^{n}$, where vertexes are placed on the coordinate axes of a Cartesian orthogonal reference frame, $\left(\mathrm{O} x_{1} x_{2} \ldots x_{n}\right)$, at a distance, $x_{i}=12,1 \leq i \leq 12$, from the origin.

The method is aimed to help musicians with basic knowledge of algebra, combinatorics and group theory, but desirous of following a line of thought to get results. This is why, say, the binomial theorem has been inferred instead of being directly used. The procedure can be generalized to musical intervals in multiples of semitones under $L$-note (instead of 12-note) equal temperament and equivalence relations other than $T_{n}, T_{n} / T_{n} I$, even if expected to be more cumbersome. Of course, group theory remains the more powerful 
tool to this respect, and the current approach should, ultimately, predispose the reader towards further deepening on this subject.

According to Eqs. (5) and (28), the number of $T_{n}$ set classes of cardinality, $n$, reads:

$$
\begin{aligned}
\nu_{\mathrm{M}}(n) & =\frac{N_{\mathrm{M}}(n)}{n}=\frac{N_{\mathrm{C}}(n)+\Delta N(n)}{n} \\
& =\frac{1}{12}\left(\begin{array}{c}
12 \\
n
\end{array}\right)+\frac{1}{n} \sum_{i=1}^{12} \zeta(12 i, n)(n-i) \nu_{i}(n) ;
\end{aligned}
$$

where $\zeta\left(m_{1}, m_{2}\right)$ is defined by Eq. (19) and $\nu_{i}(n)$ is listed in Table 3, keeping in mind $i>n$ and/or $n>5$ implies $\nu_{i}(n)=0$.

Within the framework of group theory, the result is e.g., [15] $\S 72$ :

$$
\nu_{\mathrm{M}}(n)=\frac{1}{12} \sum_{j=1}^{12} \zeta(12, j) \zeta(n, j) \phi(j)\left(\begin{array}{c}
12 / j \\
n / j
\end{array}\right) ;
$$

where $\phi(k)$ is the Euler phi function e.g., [14] Chap. $9 \S 9.7$ and the product, $\zeta(12, j) \zeta(n, j)$, by definition equals unity provided $j$ is a common divisor of $n$ and 12 , and equals zero otherwise. Keeping in mind $\phi(1)=1$ e.g., 23 . $\S 24.3 .2$, Eq. (70) may be cast uder the form:

$$
\nu_{\mathrm{M}}(n)=\frac{1}{12}\left(\begin{array}{c}
12 \\
n
\end{array}\right)+\frac{1}{12} \sum_{j=2}^{12} \zeta(12, j) \zeta(n, j) \phi(j)\left(\begin{array}{c}
12 / j \\
n / j
\end{array}\right) ;
$$

where the first term on the right-hand side of Eq. (171) equals its counterpart in Eq. (69) that is, via Eq. (77), the fractional number, $N_{\mathrm{C}} / n$, of distinct $n$ chords. Then the second term on the right-hand side of Eq. (71) equals the fractional number, $\Delta N / n$, of repeating $n$-chords, as listed in Table 6 .

Leaving aside the special case of null cardinality, $n=0$, an inspection of Table 6] shows the terms of the sum on the right-hand side of Eqs. (711) and (69), respectively, coincide with the exception of $n=4,6,8$, even if an equal result is attained, which implies related formulations are not intrinsically equivalent.

For $n=0, \lim _{n \rightarrow 0^{+}} \Delta N(n) / n=11 / 12$ is inferred from Table 1 via Eqs. (14) and (69). On the other hand, $(1+2+2+2+4) / 12=11 / 12$ via Eq. (71), in agreement with the results listed in Table 6.

A similar comparison could be performed with regard to $T_{n} / T_{n} I$ set classes by use of the counterparts of Eqs. (69) and (70) e.g., [15] $\S 72$ which implies a more complex formulation and for this reason it has not been considered. 
Table 6: Fractional number of repeating $n$-chords, $\Delta N / n$, of each cardinality, $n, 1 \leq n \leq 12$, inferred from group theory (GT), Eq. (71), and from the current paper (CP), Eq. (69). For $n=4,6,8$, related addends are different even if the sum yields equal results. For $n=0$, Eq. (14) has also been used. See text for further details.

\begin{tabular}{|r|r|c|c|}
\hline \hline$n$ & $\phi$ & $\Delta N / n(\mathrm{GT})$ & $\Delta N / n(\mathrm{CP})$ \\
0 & 1 & $11 / 12$ & $11 / 12$ \\
1 & 1 & $0 / 12$ & $0 / 1$ \\
2 & 1 & $6 / 12$ & $1 / 2$ \\
3 & 2 & $8 / 12$ & $2 / 3$ \\
4 & 2 & $(15+6) / 12$ & $(3+4) / 4$ \\
5 & 4 & $0 / 12$ & $0 / 5$ \\
6 & 2 & $(20+12+4) / 12$ & $(5+4+9) / 6$ \\
7 & 6 & $0 / 12$ & $0 / 7$ \\
8 & 4 & $(15+6) / 12$ & $(6+8) / 8$ \\
9 & 6 & $8 / 12$ & $6 / 9$ \\
10 & 4 & $6 / 12$ & $5 / 10$ \\
11 & 10 & $0 / 12$ & $0 / 11$ \\
12 & 4 & $11 / 12$ & $11 / 12$ \\
\hline
\end{tabular}


Symmetries found in enumerating $n$-chords, as shown by results listed in Tables 1, 2, 3, are intrinsic to the geometrical interpretation. More specifically, $n$-hedrons exhibit $(j-1)$-faces and $(n-j-1)$-faces, $1 \leq j \leq n-1$, in equal number, as listed in Table 7, which can be expressed via binomial coefficients. In addition, the boundary condition expressed by Eq. (1) implies regular, inclined $n$-hedrons, $\Psi_{12, i}^{n}$, where vertexes lie on the coordinate axes at a distance, $x_{i}=12,1 \leq i \leq n$, from the origin.

It is worth emphasizing symmetries are intrinsic to $n$-hedrons and, for this reason, can be described using the binomial formula. In particular, regular inclined $n$-hedrons, $\Psi_{12}^{n}$, can be partitioned into $n$ congruent $n$-hedrons, $\Psi_{12, i}^{n}$, $1 \leq i \leq n$, by joining the orthocentre with each vertex. Different $\Psi_{12, i}^{n}$ have in common $(n-2)$-faces where a vertex is the orthocentre of $\Psi_{12}^{n}$.

All of $n$-chords belonging to an assigned $T_{n}$ or $T_{n} / T_{n} I$ set class are similarly or similarly and symmetrically placed, respectively, within different $\Psi_{12, i}^{n}$, where distinct $n$-chords relate to positive integer points belonging to $\Psi_{12, i}^{n}, 1 \leq i \leq n$, while repeating $n$-chords, if any, are placed on $(n-2)$-faces of $\Psi_{12, i}^{n}$ where a vertex is the orthocentre of $\Psi_{12}^{n}$.

The geometric representation exploited in the current paper looks appealing, in that the idea is very simple and very effective, which is always a good point. The fact that the dimension of the $(n-1)$-plane where $n$-chords are located only depends on the scale size, and not on the chromatic gamut in which the scale is embedded (it only affects the number of points considered) makes it very useful. One could for example easily visualise triads in quarter-tone scales, or on even finer-grained scales, on an ordinary (2-) plane.

Last but not least, the above mentioned geometric representation has musical significance: rotations around the $n$-sector (i.e. an axis normal to the $(n-1)$-plane through the orthocentre of $\left.\Psi_{12}^{n}\right)$ correspond to chord inversions (in musical language), or cyclic permutations (in mathematical language). Looking at their location tells if a scale may have internal symmetries (repeating chords). Similarly, rotations about an axis joining orthocentres of opposite $(j-1)$ and $(n-1-j)$-faces correspond to chord reflections, which are symmetrically placed with respect to the rotation axis. But surely there are many more which would be worth investigating further.

Enumeration of $n$-chords within an algebraic or geometrical framework yields coinciding results but, in the latter alternative, further perspectives can be exploited such as counting integer points within lattice polytopes.

Following a similar line of thought, the results of the current paper could be extended to musical intervals in multiples of semitones under $L$-note equal temperament, reproducing results found within the framework of group theory e.g., [17] [15].

The group theory, of course, remains the more powerful tool in dealing 
with any kind of equivalence relation, but further insight on specific problems could be gained in the light of both algebraic and geometric method outlined in the current paper.

More specifically, using group theory in counting specified pitch-class sets could be related to the description of a high-population $(N \gg 1)$ statistical system e.g., a perfect gas within a box, in terms of observables e.g., volume, pressure, temperature. Conversely, the enumeration of pitch-class sets within an algebraic or geometric framework, as outlined in the current paper, could be related to the description of a low-population $(N \gtrsim 1)$ statistical system e.g., perfectly elastic dissipationless spheres moving on a horizontal plane bounded by perfectly elastic dissipationless walls, in terms of integration of the motion equations from the knowledge of initial conditions.

\section{Conclusion}

The current investigation is restricted to musical intervals in multiples of semitones under 12-note equal temperament, in particular about the question of how many $n$-chords there are of a given cardinality, and how many $T_{n}$ or $T_{n} / T_{n} I$ set classes. In the light of a geometrical interpretation, $n$-chords of a given cardinality, $\left\{\ell_{1}, \ell_{2}, \ldots, \ell_{n}\right\}$, satisfying the boundary condition expressed by Eq. (1), are related to positive integer points in $\Re^{n}$, belonging to a regular inclined $n$-hedron, $\Psi_{12}^{n}$. Joining vertexes with the orthocentre yields $n$ congruent $n$-hedrons, $\Psi_{12, i}^{n}, 1 \leq i \leq n$, where points with coordinates related by circular permutations i.e. the $n$ elements of $T_{n}$ set classes, are equally partitioned and similarly placed. If $T_{n}$ set classes exhibit repeating $n$-chords, then related points are lying on $(n-2)$-faces of $\Psi_{12, i}^{n}$ where a vertex is the orthocentre of $\Psi_{12}^{n}$.

The number, $N_{\mathrm{C}}(n)$, of distinct $n$-chords is determined within an algebraic framework, and a symmetry is found around $q=13 / 2$ as listed in Table 1. The fractional number, $N_{\mathrm{C}}(n) / n$, of distinct $n$-chords shows a symmetry around $n=6$, as listed in Table 1, provided the domain is extended to $n=0$ in connection with Euclidean 0 -spaces, $\Re^{0}$. The fractional numbers, $\Delta N(n) / n$ and $N_{\mathrm{M}}(n) / n=\left[N_{\mathrm{C}}(n)+\Delta N(n)\right] / n$, of repeating and total (distinct + repeating) $n$-chords, respectively, also exhibit a symmetry around $n=6$, including $n=0$, as listed in Table 11. It is worth remembering $\nu_{\mathrm{M}}(n)=N_{\mathrm{M}}(n) / n$ is the number of $T_{n}$ set classes.

Following the same line of thought, the above results can be generalized to musical intervals in multiples of semitones under $L$-note equal temperament.

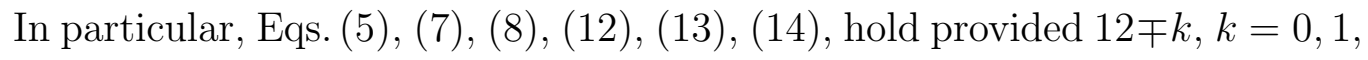
is replaced by $L \mp k$ therein. 
The above mentioned symmetries are disclosed within a geometrical framework, where $n$-chords together with $T_{n}$ and $T_{n} / T_{n} I$ set classes can be enumerated as well. More specifically, symmetries are intrinsic to $n$-hedrons, in general on one hand, and via the boundary condition of the problem on the other hand.

The main results of the current paper can be summarized as follows.

(1) Using a one-to-one correspondence between $n$-chords and positive integer points within an Euclidean $n$-space, $\Re^{n}$, both distinct and repeating $n$-chords are enumerated within the framework of an algebraic method together with $T_{n}$ and $T_{n} / T_{n} I$ set classes. Related results equal their counterparts already known in the context of group theory e.g., [17] [14] Chap. 9 [15].

(2) Positive integer points related to $n$-chords of cardinality, $n$, belong to a regular inclined $n$-hedron, $\Psi_{12}^{n}$, of vertexes, $\mathrm{V}_{\mathrm{i}} \equiv\left(12 \delta_{1 i}, 12 \delta_{2 i}, \ldots, 12 \delta_{n i}\right)$, $1 \leq i \leq n$. By joining the orthocentre, $\mathrm{H}_{\mathrm{n}} \equiv(12 / n, 12 / n, \ldots, 12 / n)$, with vertexes, $\mathrm{V}_{\mathrm{i}}, \Psi_{12}^{n}$ can be subdivided into $n$ congruent $n$-hedrons, $\Psi_{12, i}^{n}, 1 \leq$ $i \leq n$. Positive integer points related to $n$-chords within an assigned $T_{n}$ or $T_{n} / T_{n} I$ set class are equally partitioned among and similarly or similarly and symmetrically placed within $\Psi_{12, i}^{n}$. In particular, points related to repeating $n$-chords are placed on $(n-2)$-faces between different $\Psi_{12, i}^{n}$, to be counted according to their multiplicity i.e. the number of involved $\Psi_{12, i}^{n}$.

(3) Both distinct and repeating $n$-chords are enumerated within the framework of a geometrical method together with $T_{n}$ and $T_{n} / T_{n} I$ set classes, reproducing their counterparts inferred from an algebraic method in (1).

(4) Results in (3) allow the calculation of nonnegative integer (i.e. exhibiting nonnegative integer coordinates) points belonging to $\Psi_{12}^{n}$ which, to this respect, can be considered as special cases of lattice polytopes in $\Re^{n}$.

(5) Symmetries shown by the number of $n$-chords and $T_{n}$ or $T_{n} / T_{n} I$ set classes with respect to the cardinality, $n=6$, are intrinsic to the geometry in a twofold manner. In general, the number of $(j-1)$-faces of $n$-hedrons, $1 \leq j \leq n-1$, can be expressed via binomial coefficients as listed in Table 7 . In particular, the boundary condition of the problem, expressed by Eq. (1), implies consideration of regular inclined $n$-hedrons, $\Psi_{12}^{n}$, where the number of positive integer points can also be expressed via the binomial formula, as shown in Table 1. To this respect, $\Re^{n}$ and $\Re^{12-n}, 0 \leq n \leq 12$, or $\Re^{6-m}$, $\Re^{6+m}, 0 \leq m \leq 6$, can be considered as Euclidean complementary spaces. More generally, the symmetry of the results inferred from the group theory could be conceived as intrinsic to lattice polytopes in $\Re^{n}$. 


\section{Acknowledgements}

The authors are indebted to two anonymous referees of an earlier version of the paper (2008), for critical comments and useful suggestions.

\section{References}

[1] A. Loquin, Algebre de l'harmonie, Richault \& C., Paris, 1884.

[2] E. Bacon, Our musical idiom, The Open Court Publishing Company, Chicago, 1917.

[3] J. Rahn, Basic Atonal Theory, Longman, New York, 1980.

[4] R. Morris, Composition with Pitch-Classes: a Theory of Compositional Design, Yale University Press, New Haven, 1987.

[5] A. Vieru, Book of Modes, Editura Muzicala a Uniunii Compozitorilor si Muzicologilor din România, Bucarest, 1993.

[6] A. Kerber, Algebraic Combinatorics via Finite Group Actions, B.I. Wissenschaftsverlag, Mannheim, 1991.

[7] A. Kerber, Applied Finite Group Actions, Vol. 19 of Algorithms and Combinatorics, Springer, Berlin, 1999.

[8] D.L. Reiner, Enumeration in music theory, American Mathematical Monthly, 92, 51-54, 1985.

[9] A. Forte, The Structure of Atonal Music, Yale University Press, New Haven, 1973.

[10] R. Morris, Class Notes for Atonal Music Theory, Prog Peak Music, Lebanon, New Haven, 1991.

[11] J.N. Straus, Introduction to Post-Tonal Theory, Upper Saddle River, Pearson Prentice Hall, New Jersey, 2005.

[12] O. Messiaen, Technique de mon langage musical, Leduc, Paris, 1944.

[13] R. Cohn, Properties and Generability of Transpositionally Invariant Sets, Journal of Music Theory, 35, 1-32, 1991.

[14] D. Benson, Music: a Mathematical Offering, Cambridge University Press, Cambridge, 2006. 
[15] J. Hook, Why Are There Twenty-Nine Tetrachords? A Tutorial on Combinatorics and Enumeration in $\mathrm{Mu}-$ sic Theory, Music Theory Online, 13, 1-17, 2007. http://www.mtosmt.org/issues/mto.07.13.4/mto.07.13.4.hook.html

[16] H. Fripertinger, Endliche Gruppenaktionen auf Funktionenmengen. Das Lemma von Burnside - Repräsentantenkonstruktionen - Anwendungen in der Musiktheorie, Bayreuther Mathematische Schriften, 45, 19-135, 1993.

[17] H. Fripertinger, Enumeration and Construction in Music Theory, in Diderot Forum on Mathematics and Music: Computational and Mathematical Methods in Music, ed. H. G. Feichtinger and M. Dörfler, Österreichische Computergesellschaft, Vienna, 179-204, 1999. http://www.uni-graz.at/ fripert/publications.html

[18] R.C. Read, Combinatorial Problems in the Theory of Music, Discrete Mathematics, 167-168, 543-551, 1997.

[19] F. Jedrzejewski, Mathematical Theory of Music, Editions Delatour, Paris, 2006.

[20] R. Crisman, Describing Structural Aspects of Pitch-Sets Using Successive-Interval Arrays, The Journal of Music Theory, 27, 181-201, 1979.

[21] M.R. Spiegel, Mathematical Handbook of Formulas and Tables, Schaum's Outline Series, McGraw-Hill Book Company, New York, 1968.

[22] A. Barvinok, J.E. Pommersheim, An Algorithmic theory of Lattice Points in Polyedra, New Perspectives in Geometric Combinatorics, 38, 91-147, 1999.

[23] M. Abramowitz, I.A. Stegun (Eds.), The Euler Totient Function, in "Handbook of Mathematical Functions with Formulas, Graphs, and Mathematical Tables, 9th printing, p. 826, Dover, New York, 1972.

[24] R. Caimmi, Il Problema della Misura, Diade, Nuova Vita, Padova, 2000.

[25] R. Caimmi, The Arithmetic Mean Standard Deviation Distribution: A Geometrical Framework, Applied Mathematics, 4, 1-10, 2013.

[26] R. Caimmi, The Weighted Mean Standard Deviation Distribution: A Geometrical Framework, Applied Mathematics, 6, 520-546, 2015.

[27] R. Caimmi, Il Problema della Misura, Aracne, Ariccia, 2016. 


\section{Appendix}

\section{A Birthday-cake problem}

Let $N$ candles of $L$ different colour be put at equal distance from the symmetry axis of a circular birthday cake, on the $N$ vertexes of a regular polygon. Accordingly, a generic configuration is defined by a $N$-tuple of colours, selected among the available $L$, in brief a colour set. Then $T_{n}$ set classes are defined by $N$ repeated rotations of the cake about its axis by an angle, $\alpha_{\mathrm{N}}=2 \pi / N$.

The problem is: how many colour sets and $T_{n}$ set classes do exist? For sake of clarity, let attention be restricted to a simple case, $N=4$ and $L=3$ : blue $(B)$, green $(G)$, red $(R)$, say e.g., [15] $§ 46$.

A solution to the problem shall be exploited along the following steps.

(i) Calculate the total number of distinct colour sets, 4-tuples in the case under consideration.

(ii) Select different kinds of colour sets with regard to the elements of the 4-tuples.

(iii) For each kind of colour sets infer the number of repeating 4-tuples within related $T_{n}$ set classes.

(iv) Calculate the total number of distinct + repeating colour sets.

(v) Calculate the total number of $T_{n}$ set classes.

Different configurations of the birthday cake can be related to different colour sets. For each place of a 4-tuple, there are three possibilities for the choice of a colour $(\mathrm{B}, \mathrm{G}, \mathrm{R})$, yielding a total of $3^{4}=81$ distinct 4 -tuples. In addition, the following types of colour sets can be defined: $x^{4}$, where all colours are identical; $x^{3} y$, where three colours are identical and the remaining one is different; $x^{2} y^{2}$, where two different colours are identical in pairs; $x^{2} y z$, where two colours are identical and the remaining two are different. In general, $x=\mathrm{B}, \mathrm{G}, \mathrm{R} ; x \neq y \neq z$.

For $x^{4}$ colour sets, $\{x, x, x, x\}$, the number of distinct 4 -tuples is $4 ! /(4 \cdot 3$. $2)=1$, and the number of different elements, $x$, equals $\left(\begin{array}{l}3 \\ 1\end{array}\right)=3$. Accordingly, the total number of distinct and repeating 4 -tuples is $3 \cdot 1=3$ and $3 \cdot 3=9$, respectively. Then the total number of distinct + repeating colour sets is $3+9=12$.

For $x^{3} y$ colour sets, $\{x, x, x, y\}$, the number of distinct 4 -tuples is $4 ! /(3$. $2)=4$, and the number of different pairs, $x y$, equals $\left(\begin{array}{l}4 \\ 2\end{array}\right)=6$. Accordingly, the total number of distinct and repeating 4 -tuples is $6 \cdot 4=24$ and $0 \cdot 4=0$, 
respectively. Then the total number of distinct + repeating colour sets is $24+0=24$.

For $x^{2} y^{2}$ colour sets, $\{x, x, y, y\}$, the number of distinct 4 -tuples is $4 ! /(2$. $2)=6$, and the number of different pairs, $x y$, equals $\left(\begin{array}{l}3 \\ 2\end{array}\right)=3$. Accordingly, the total number of distinct and repeating 4-tuples is $3 \cdot 6=18$ and $3 \cdot(1+1)=$ 6 , respectively, the last due to $\{x, y, x, y\}$ and $\{y, x, y, x\}$. Then the total number of distinct + repeating colour sets is $18+6=24$.

For $x^{2} y z$ colour sets, $\{x, x, y, z\}$, the number of distinct 4 -tuples is $4 ! / 2=$ 12 , and the number of different triads, $x y z$, equals $\left(\begin{array}{l}3 \\ 1\end{array}\right)=3$. Accordingly, the total number of distinct and repeating 4-tuples is $3 \cdot 12=36$ and $0 \cdot 12=0$, respectively. Then the total number of distinct + repeating colour sets is $36+0=36$.

More specifically, in the case under discussion the total number of colour sets belonging to an assigned type can be determined along the following steps.

(a) Start from an assigned $T_{n}$ set class of the type considered.

(b) Exchange $y$ with $z$ (if both present) to get a distinct (if any) $T_{n}$ set class.

(c) Exchange $x$ with $y$ (only once in case of multiplicity) to get a distinct (if any) $T_{n}$ set class.

For $x^{4}$ colour sets, the application of the method yields:

$$
\{x, x, x, x\},\{x, x, x, x\},\{x, x, x, x\},\{x, x, x, x\} ;
$$

where no additional exchange has to be performed as both $y$ and $z$ are absent, yielding $1 T_{n}$ set class made of 1 distinct and 3 repeating colour sets. Keeping in mind $x=\mathrm{B}, \mathrm{G}, \mathrm{R}$, there are $3 \cdot 1=3 T_{n}$ set classes for a total of $1 \cdot 3=3$ distinct and $3 \cdot 3=9$ repeating colour sets, according to the above results.

For $x^{3} y$ colour sets, the application of the method yields:

$$
\{x, x, x, y\},\{x, x, y, x\},\{x, y, x, x\},\{y, x, x, x\} ;
$$

where no additional exchange has to be performed as $z$ is absent, yielding $1 T_{n}$ set class made of 4 distinct colour sets. Keeping in mind $x y=$ BG, GR, RB, GB, RG, BR, $(x y \neq y x$ due to different multiplicities in $x$ and $y$ ), there are $6 \cdot 1=6 T_{n}$ set classes for a total of $6 \cdot 4=24$ distinct and $0 \cdot 4=0$ repeating colour sets, according to the above results.

For $x^{2} y^{2}$ colour sets, the application of the method yields:

$$
\begin{aligned}
& \{x, y, y, x\},\{y, y, x, x\},\{y, x, x, y\},\{x, x, y, y\} \\
& \{x, y, x, y\},\{y, x, y, x\},\{x, y, x, y\},\{y, x, y, x\}
\end{aligned}
$$


where no additional exchange has to be performed as $z$ is absent, yielding 2 $T_{n}$ set classes made of $(4+2)$ distinct and 2 repeating colour sets. Keeping in mind $x y=\mathrm{BG}, \mathrm{GR}, \mathrm{RB},(x y=y x$ do to equal multiplicities in $x$ and $y)$, there are $3 \cdot 2=6 T_{n}$ set classes for a total of $3 \cdot(4+2)=18$ distinct and $3 \cdot 2=6$ repeating colour sets, according to the above results.

For $x^{2} y z$ colour sets, the application of the method yields:

$$
\begin{aligned}
& \{x, x, y, z\},\{x, y, z, x\},\{y, z, x, x\},\{z, x, x, y\} ; \\
& \{x, x, z, y\},\{x, z, y, x\},\{z, y, x, x\},\{y, x, x, z\} ; \\
& \{x, y, x, z\},\{y, x, z, x\},\{x, z, x, y\},\{z, x, y, x\} ;
\end{aligned}
$$

where no additional exchange has to be performed, yielding $3 T_{n}$ set classes made of $3 \cdot 4=12$ distinct colour sets. Keeping in mind $x=\mathrm{B}, \mathrm{G}, \mathrm{R}$; $x \neq y \neq z$; there are $3 \cdot 3=9 T_{n}$ set classes for a total of $3 \cdot 12=36$ distinct and $0 \cdot 12=0$ repeating colour sets, according to the above results.

Finally, the number of distinct, $N_{\mathrm{C}}$, repeating, $\Delta N$, total, $N_{\mathrm{M}}$, colour sets, respectively, reads:

$$
\begin{aligned}
& \begin{aligned}
N_{\mathrm{C}}=N_{\mathrm{C}}\left(x^{4}\right)+N_{\mathrm{C}}\left(x^{3} y\right)+N_{\mathrm{C}}\left(x^{2} y^{2}\right)+N_{\mathrm{C}}\left(x^{2} y z\right)=3+24+18+36 \\
=81
\end{aligned} \\
& \begin{aligned}
\Delta N=\Delta N\left(x^{4}\right)+\Delta N\left(x^{3} y\right)+\Delta N\left(x^{2} y^{2}\right)+\Delta N\left(x^{2} y z\right)=9+0+6+0 \\
=15 ;
\end{aligned} \\
& \begin{aligned}
N_{\mathrm{M}}=N_{\mathrm{C}}+\Delta N=12+24+24+36=96 ;
\end{aligned}
\end{aligned}
$$

and the number of $T_{n}$ set classes, $\nu_{\mathrm{M}}$, is:

$$
\nu_{\mathrm{M}}=\frac{N_{\mathrm{M}}}{4}=\frac{12}{4}+\frac{24}{4}+\frac{24}{4}+\frac{36}{4}=3+6+6+9=24 ;
$$

or $96 / 4=24$.

Within the framework of group theory, the application of Burnside's lemma to birthday-cake problem yields e.g., [15] $§ 46$ :

$$
\nu_{\mathrm{M}}=\frac{81}{4}+\frac{3}{4}+\frac{9}{4}+\frac{3}{4}=\frac{96}{4}=24 ;
$$

which is in agreement with Eq. (75) but the addends are different.

Within the framework of group theory, the application of Polya's theorem to birthday-cake problem yields e.g., [15] $§ 62$ :

$$
\begin{aligned}
\nu_{\mathrm{M}} & =(1+1+1)+(1+1+1+1+1+1)+2(1+1+1)+3(1+1+1) \\
& =3+6+6+9=24
\end{aligned}
$$


which is in agreement with Eq. (75), exhibiting equal addends.

The result from Eq. (76) is based on Burnside's lemma and does not involve weights on $T_{n}$ set classes. On the other hand, the weighted method given by Polya's theorem is simply a refinement of the same idea, showing exactly how the $24 T_{n}$ set classes are distributed among the various weights e.g., [15] §63. For further details, an interested reader is addressed to specialized monographs e.g., [15] or textbooks e.g., [14] Chap.9. The current method exactly reproduces the results inferred via Polya's theorem.

\section{B Necklace problem}

Let $N$ beads of $L$ different colour be put at equal distance from the symmetry axis of a circular necklace, on the $N$ vertexes of a regular polygon. Accordingly, a generic configuration is defined by a $N$-tuple of colours, selected among the available $L$, in brief a colour set. Then $T_{n}$ set classes are defined by $N$ repeated rotations of the necklace about its axis by an angle, $\alpha_{\mathrm{N}}=2 \pi / N ; T_{n} I$ set classes are similarly defined, but with the addition of flipping over; $T_{n} / T_{n} I$ set classes are the union of pairwise $T_{n}$ and $T_{n} I$ set classes. The problem is: how many colour sets and $T_{n} / T_{n} I$ set classes do exist? For sake of clarity, let attention be restricted to a simple case, $N=4$ and $L=3$ : blue (B), green $(\mathrm{G})$, red $(\mathrm{R})$, say e.g., [15] $\S 47$.

With regard to distinct colour sets and $T_{n}$ set classes, the results coincide with their counterparts of the birthday-cake problem, where the total number equals 81 and 24, respectively. The total number of $T_{n} I$ set classes, by definition, also equals 24 . What remains to be done is the enumeration of repeating colour sets and $T_{n} / T_{n} I$ set classes.

Among the eight colour sets within a generic $T_{n} / T_{n} I$ set class, two quadruplets exhibit colour sets related via circular permutation and, in addition, four doublets related via reflection. Accordingly, palindrome and pseudo palindrome colour sets must be counted as repeating. More specifically, $T_{n}$ and $T_{n} I$ i.e. pairwise $T_{n}$ set classes made of distinct colour sets yield a single $T_{n} / T_{n} I$ set class, while single $T_{n}$ set classes made of palindrome or pseudo palindrome colour sets yield a single $T_{n} / T_{n} I$ set class.

For $x^{4}$ colour sets, $\{x, x, x, x\}$, the number of distinct 4 -tuples is $4 ! /(4 \cdot 3$. $2)=1$, and the number of different elements, $x$, equals $\left(\begin{array}{l}3 \\ 1\end{array}\right)=3$. Accordingly, the total number of distinct and repeating 4 -tuples is $3 \cdot 1=3$ and $3 \cdot 3=9$, respectively. But colour sets are palindrome in the case under discussion, which implies 4 additional 4 -tuples for a total of $3 \cdot(3+4)=21$ repeating colour sets. Then the total number of distinct + repeating colour sets is $3+21=24$. 
For $x^{3} y$ colour sets, $\{x, x, x, y\}$, the number of distinct 4 -tuples is $4 ! /(3$. $2)=4$, and the number of different pairs, $x y$, equals $\left(\begin{array}{l}4 \\ 2\end{array}\right)=6$. Accordingly, the total number of distinct and repeating 4-tuples is $6 \cdot 4=24$ and $0 \cdot 4=$ 0 , respectively. But colour sets are pseudo palindrome in the case under discussion, which implies 4 additional 4 -tuples for a total of $6 \cdot(0+4)=24$ repeating colour sets. Then the total number of distinct + repeating colour sets is $24+24=48$.

For $x^{2} y^{2}$ colour sets, $\{x, x, y, y\}$, the number of distinct 4-tuples is $4 ! /(2$. $2)=6$, and the number of different pairs, $x y$, equals $\left(\begin{array}{l}3 \\ 2\end{array}\right)=3$. Accordingly, the total number of distinct and repeating 4 -tuples is $3 \cdot 6=18$ and $3 \cdot(1+1)=$ 6 , respectively, the last due to $\{x, y, x, y\}$ and $\{y, x, y, x\}$. But colour sets are palindrome or pseudo palindrome in the case under discussion, which implies $4+4=8$ additional 4 -tuples for a total of $3 \cdot(2+8)=30$ repeating colour sets. Then the total number of distinct + repeating colour sets is $18+30=$ 48.

For $x^{2} y z$ colour sets, $\{x, x, y, z\}$, the number of distinct 4-tuples is $4 ! / 2=$ 12 , and the number of different triads, $x y z$, equals $\left(\begin{array}{l}3 \\ 1\end{array}\right)=3$. Accordingly, the total number of distinct and repeating 4-tuples is $3 \cdot 12=36$ and $0 \cdot 12=0$, respectively. But colour sets, $\{x, y, x, z\}$, are pseudo palindrome in the case under discussion, which implies 4 additional 4 -tuples for a total of $3 \cdot(0+4)=$ 12 repeating colour sets. Then the total number of distinct + repeating colour sets is $36+12=48$.

More specifically, in the case under discussion the total number of colour sets belonging to an assigned kind can be determined along the following steps.

(a) Start from an assigned $T_{n} / T_{n} I$ set class of the kind considered.

(b) Exchange $y$ with $z$ (if both present) to get a distinct (if any) $T_{n} / T_{n} I$ set class.

(c) Exchange $x$ with $y$ (only once in case of multiplicity) to get a distinct (if any) $T_{n} / T_{n} I$ set class.

For $x^{4}$ colour sets, the application of the method yields:

$$
\begin{aligned}
& \{x, x, x, x\},\{x, x, x, x\},\{x, x, x, x\},\{x, x, x, x\} \\
& \{x, x, x, x\},\{x, x, x, x\},\{x, x, x, x\},\{x, x, x, x\}
\end{aligned}
$$

where no additional exchange has to be performed as both $y$ and $z$ are absent, yielding $1 T_{n} / T_{n} I$ set class made of 1 distinct and $3+4=7$ repeating colour sets. Keeping in mind $x=\mathrm{B}, \mathrm{G}, \mathrm{R}$, there are $3 \cdot 1=3 T_{n} / T_{n} I$ set classes for 
a total of $1 \cdot 3=3$ distinct and $7 \cdot 3=21$ repeating colour sets, according to the above results.

For $x^{3} y$ colour sets, the application of the method yields:

$$
\begin{aligned}
& \{x, x, x, y\},\{x, x, y, x\},\{x, y, x, x\},\{y, x, x, x\} \\
& \{y, x, x, x\},\{x, y, x, x\},\{x, x, y, x\},\{x, x, x, y\}
\end{aligned}
$$

where no additional exchange has to be performed as $z$ is absent, yielding 1 $T_{n} / T_{n} I$ set class made of 4 distinct and 4 repeating colour sets. Keeping in mind $x y=\mathrm{BG}, \mathrm{GR}, \mathrm{RB}, \mathrm{GB}, \mathrm{RG}, \mathrm{BR},(x y \neq y x$ due to different occurrences in $x$ and $y$ ), there are $6 \cdot 1=6 T_{n} / T_{n} I$ set classes for a total of $6 \cdot 4=24$ distinct and $6 \cdot 4=24$ repeating colour sets, according to the above results.

For $x^{2} y^{2}$ colour sets, the application of the method yields:

$$
\begin{aligned}
& \{x, y, y, x\},\{y, y, x, x\},\{y, x, x, y\},\{x, x, y, y\} \\
& \{x, y, y, x\},\{x, x, y, y\},\{y, x, x, y\},\{y, y, x, x\} ; \\
& \{x, y, x, y\},\{y, x, y, x\},\{x, y, x, y\},\{y, x, y, x\} ; \\
& \{y, x, y, x\},\{x, y, x, y\},\{y, x, y, x\},\{x, y, x, y\} ;
\end{aligned} ;
$$

where no additional exchange has to be performed as $z$ is absent, yielding 2 $T_{n} / T_{n} I$ set classes made of $4+2=6$ distinct and $4+6=10$ repeating colour sets. Keeping in mind $x y=\mathrm{BG}, \mathrm{GR}, \mathrm{RB},(x y=y x$ due to equal occurrences in $x$ and $y)$, there are $3 \cdot 2=6 T_{n} / T_{n} I$ set classes for a total of $3 \cdot(4+2)=18$ distinct and $3 \cdot(4+6)=30$ repeating colour sets, according to the above results.

For $x^{2} y z$ colour sets, the application of the method yields:

$$
\begin{aligned}
& \{x, x, y, z\},\{x, y, z, x\},\{y, z, x, x\},\{z, x, x, y\} \\
& \{z, y, x, x\},\{x, z, y, x\},\{x, x, z, y\},\{y, x, x, z\} ; \\
& \{x, x, z, y\},\{x, z, y, x\},\{z, y, x, x\},\{y, x, x, z\} \\
& \{y, z, x, x\},\{x, y, z, x\},\{x, x, y, z\},\{z, x, x, y\} ; \\
& \{x, y, x, z\},\{y, x, z, x\},\{x, z, x, y\},\{z, x, y, x\} \\
& \{z, x, y, x\},\{x, z, x, y\},\{y, x, z, x\},\{x, y, x, z\} ;
\end{aligned} ;
$$

where no additional exchange has to be performed, yielding $2 T_{n} / T_{n} I$ set classes (the first two coincide) made of $3 \cdot 4=12$ distinct and $1 \cdot 4=4$ repeating colour sets. Keeping in mind $x=\mathrm{B}, \mathrm{G}, \mathrm{R}, x \neq y \neq z$, there are $3 \cdot 2=6 T_{n} / T_{n} I$ set classes for a total of $3 \cdot 12=36$ distinct and $3 \cdot 4=12$ repeating colour sets, according to the above results. 
Finally, the total number of distinct, $N_{\mathrm{C}}$, repeating, $\Delta N$, and total, $N_{\mathrm{M}}$, colour sets, respectively, reads:

$$
\begin{aligned}
& \begin{aligned}
N_{\mathrm{C}}=N_{\mathrm{C}}\left(x^{4}\right)+N_{\mathrm{C}}\left(x^{3} y\right)+N_{\mathrm{C}}\left(x^{2} y^{2}\right)+N_{\mathrm{C}}\left(x^{2} y z\right)=3 & +24+18+36 \\
= & 81
\end{aligned} \\
& \begin{aligned}
\Delta N=\Delta N\left(x^{4}\right)+\Delta N\left(x^{3} y\right)+\Delta N\left(x^{2} y^{2}\right)+\Delta N\left(x^{2} y z\right) & =21+24+30+12 \\
& =87
\end{aligned} \\
& N_{\mathrm{M}}=N_{\mathrm{C}}+\Delta N=24+48+48+48=168 ;
\end{aligned}
$$

and the number of $T_{n} / T_{n} I$ set classes, $\nu_{\mathrm{M}}$, is:

$$
\nu_{\mathrm{M}}=\frac{N_{\mathrm{M}}}{8}=\frac{24}{8}+\frac{48}{8}+\frac{48}{8}+\frac{48}{8}=3+6+6+6=21 ;
$$

or $168 / 8=21$.

Within the framework of group theory, the application of Burnside's lemma to necklace problem yields e.g., [15] §47:

$$
\nu_{\mathrm{M}}=\frac{84}{8}+\frac{12}{8}+\frac{36}{8}+\frac{36}{8}=\frac{168}{8}=21 ;
$$

which is in agreement with Eq. (81) but the addends are different.

Within the framework of group theory, the application of Polya's theorem to necklace problem yields e.g., [15] §64:

$$
\begin{aligned}
\nu_{\mathrm{M}} & =(1+1+1)+(1+1+1+1+1+1)+2(1+1+1)+2(1+1+1) \\
& =3+6+6+6=21
\end{aligned}
$$

which is in agreement with Eq. (81), exhibiting equal addends.

The result from Eq. (82) is based on Burnside's lemma and does not involve weights on $T_{n} / T_{n} I$ set classes. On the other hand, the weighted method given by Polya's theorem is simply a refinement of the same idea, showing exactly how the $21 T_{n} / T_{n} I$ set classes are distributed among the various weights e.g., 15] §63. For further details, an interested reader is addressed to specialized monographs e.g., [15] or textbooks e.g., [14] Chap. 9. The current method exactly reproduces the results inferred via Polya's theorem.

\section{Basic ideas on $\Psi_{12}^{n} n$-hedrons}

\section{C.1 Analytic geometry in $\Re^{k}$}

The formulation of analytic geometry in ordinary Euclidean space, $\Re^{3}$, can easily be extended to Euclidean $k$-spaces, $\Re^{k}$. 
With regard to a Cartesian orthogonal reference frame, $\left(\mathrm{O} x_{1} x_{2}, \ldots x_{k}\right)$, let $\mathrm{P}_{\mathrm{A}} \equiv\left(x_{A 1}, x_{A 2}, \ldots, x_{A k}\right)$ and $\mathrm{P}_{\mathrm{B}} \equiv\left(x_{B 1}, x_{B 2}, \ldots, x_{B k}\right)$ be generic points. The related square distance reads:

$$
\left(\overline{\mathrm{P}_{\mathrm{A}} \mathrm{P}_{\mathrm{B}}}\right)^{2}=\sum_{i=1}^{k}\left(x_{A i}-x_{B i}\right)^{2}
$$

if $\mathrm{P}_{\mathrm{A}}$ is fixed (the origin say) while $\mathrm{P}_{\mathrm{B}}$ remains generic, then Eq. (84) represents a $k$-dimension sphere, or $k$-sphere, of radius, $R=\overline{\mathrm{P}_{\mathrm{A}} \mathrm{P}_{\mathrm{B}}}$, centered on $\mathrm{P}_{\mathrm{A}}$.

The mean point, $\mathrm{P}_{\mathrm{M}}$, of the segment joining the points, $\mathrm{P}_{\mathrm{A}}, \mathrm{P}_{\mathrm{B}}$, reads:

$$
\mathrm{P}_{\mathrm{M}} \equiv\left(\frac{x_{A 1}+x_{B 1}}{2}, \frac{x_{A 2}+x_{B 2}}{2}, \ldots, \frac{x_{A k}+x_{B k}}{2}\right) ;
$$

and the identities, $\overline{\mathrm{P}_{A} \mathrm{P}_{M}}=\overline{\mathrm{P}_{\mathrm{B}} \mathrm{P}_{\mathrm{M}}}, \overline{\mathrm{P}_{\mathrm{A}} \mathrm{P}_{\mathrm{M}}}+\overline{\mathrm{P}_{\mathrm{B}} \mathrm{P}_{\mathrm{M}}}=\overline{\mathrm{P}_{\mathrm{A}} \mathrm{P}_{\mathrm{B}}}$, can easily be verified via Eqs. (84) and (85).

Let $r, r^{\prime}$, be generic straight lines in $\Re^{k}$, expressed as:

$$
\begin{array}{ll}
r: & \frac{x_{1}-x_{10}}{L_{1}}=\frac{x_{2}-x_{20}}{L_{2}}=\ldots=\frac{x_{k}-x_{k 0}}{L_{k}} ; \\
& L_{i}=\bar{x}_{i 0}-x_{i 0} ; \quad 1 \leq i \leq k ; \\
r^{\prime}: & \frac{x_{1}^{\prime}-x_{10}^{\prime}}{L_{1}^{\prime}}=\frac{x_{2}^{\prime}-x_{20}^{\prime}}{L_{2}^{\prime}}=\ldots=\frac{x_{k}^{\prime}-x_{k 0}^{\prime}}{L_{k}^{\prime}} ; \\
& L_{i}^{\prime}=\bar{x}_{i 0}^{\prime}-x_{i 0}^{\prime} ; \quad 1 \leq i \leq k ;
\end{array}
$$

where $\mathrm{P}_{0} \equiv\left(x_{10}, x_{20}, \ldots, x_{k 0}\right), \overline{\mathrm{P}}_{0} \equiv\left(\bar{x}_{10}, \bar{x}_{20}, \ldots, \bar{x}_{k 0}\right)$, are selected points on $r$ and $\mathrm{P}_{0}^{\prime} \equiv\left(x_{10}^{\prime}, x_{20}^{\prime}, \ldots, x_{k 0}^{\prime}\right), \overline{\mathrm{P}}_{0}^{\prime} \equiv\left(\bar{x}_{10}^{\prime}, \bar{x}_{20}^{\prime}, \ldots, \bar{x}_{k 0}^{\prime}\right)$, are selected points on $r^{\prime}$.

The angle, $\widehat{r r^{\prime}}$, can be expressed as:

$$
\left|\cos \widehat{r r^{\prime}}\right|=\left|\frac{\sum_{i=1}^{k} L_{i} L_{i}^{\prime}}{\left[\sum_{i=1}^{k} L_{i}^{2}\right]^{1 / 2}\left[\sum_{i=1}^{k}\left(L_{i}^{\prime}\right)^{2}\right]^{1 / 2}}\right| ;
$$

where the orthogonality condition reads $\cos \widehat{r r^{\prime}}=0$.

Let $p$ be a generic $(k-1)$-plane in $\Re^{k}$, expressed as:

$$
p: \quad a_{1} x_{1}+a_{2} x_{2}+\ldots+a_{k} x_{k}+a_{0}=0 ;
$$

where $-a_{0} / a_{i}$, is the intercept of $p$ on the coordinate axis, $x_{i}, 1 \leq i \leq k$. 
The angle, $\widehat{r p}$, between the straight line, $r$, and the $(k-1)$-plane, $p$, can be expressed as:

$$
|\sin \widehat{r p}|=\left|\frac{\sum_{i=1}^{k} L_{i} a_{i}}{\left[\sum_{i=1}^{k} L_{i}^{2}\right]^{1 / 2}\left[\sum_{i=1}^{k} a_{i}^{2}\right]^{1 / 2}}\right| ;
$$

where the orthogonality condition reads $\sin \widehat{r p}=\mp 1$.

For further details, an interested reader is addressed to earlier investigations [24] Chap. $2 \S 2.16$ [25] [26] [27] Chap. $4 \S 4.17$.

\section{C.2 General properties of $(k+1)$-hedrons}

With regard to an Euclidean $k$-space, $\Re^{k}$, and a Cartesian orthogonal reference frame, $\left(\mathrm{O} x_{1} x_{2} \ldots x_{k}\right)$, let $(k+1) k$-misaligned points be assigned as $\mathrm{V}_{1}, \mathrm{~V}_{2}, \ldots, \mathrm{V}_{\mathrm{k}+1}$. Points are $k$-misaligned in the sense different $k$-tuples, $\left\{\mathrm{V}_{\mathrm{i}_{1}}, \mathrm{~V}_{\mathrm{i}_{2}}, \ldots, \mathrm{V}_{\mathrm{i}_{k}}\right\}, 1 \leq i_{1}<i_{2}<\ldots<i_{k} \leq k+1$, belong to different $(k-1)$ planes in $\Re^{k}$. Let each point be connected with the remaining ones by segments. Let the resulting geometrical figure be defined as $(k+1)$-hedron, and denoted as $\Phi^{k+1}$. In ordinary space, $k=3$, 4-hedrons reduce to ordinary tetrahedrons.

The above definition could appear ambiguous, in that the etymology of tetrahedron implies the existence of four faces. Keeping in mind $(k+1)$ hedrons have $k$-dimensions, it can be seen (as shown below) that the number of $(k-1)$-faces equals the number of connected points, $(k+1)$. Then etymological meaning is preserved provided $(k-1)$-faces are considered instead of ordinary (2-) faces.

Another source of ambiguity lies in the fact, that " $(k+1)$-hedrons" are already defined in ordinary space, $\Re^{3}$, concerning ordinary faces e.g., hexahedron, octahedron, dodecahedron, icosahedron. Throughout the present paper, according to the current definition, $(k+1)$-hedrons are intended as solids in $\Re^{k}$ where a generic point, $\mathrm{V}_{\mathrm{i}}, 1 \leq i \leq k+1$, is connected to the remaining ( $k$-misaligned) $k$.

Any point, $\mathrm{V}_{\mathrm{i}}, 1 \leq i \leq k+1$, is a vertex or 0 -face of $\Phi^{k+1}$. Accordingly, the number of 0 -faces equals the number of distinct 1-tuples of vertexes, $\left(\begin{array}{c}k+1 \\ 1\end{array}\right)=(k+1) / 1$ !

Any duo of vertexes, $\mathrm{V}_{\mathrm{i}} \mathrm{V}_{\mathrm{j}}, i \neq j$, yields a side or 1 -face of $\Phi^{k+1}$. Accordingly, the number of 1 -faces equals the number of distinct 2-tuples of vertexes, $\left(\begin{array}{c}k+1 \\ 2\end{array}\right)=(k+1) k / 2$ !. 
Any trio of vertexes, $\mathrm{V}_{\mathrm{i}} \mathrm{V}_{\mathrm{j}} \mathrm{V}_{\ell}, i \neq j \neq \ell$, yields a triangle or 2-face of $\Phi^{k+1}$. Accordingly, the number of 2-faces equals the number of distinct 3 -tuples of vertexes, $\left(\begin{array}{c}k+1 \\ 3\end{array}\right)=(k+1) k(k-1) / 3$ !.

Any quartet of vertexes, $\mathrm{V}_{\mathrm{i}} \mathrm{V}_{\mathrm{j}} \mathrm{V}_{\ell} \mathrm{V}_{\mathrm{m}}, i \neq j \neq \ell \neq m$, yields a tetrahedron or 3-face of $\Phi^{k+1}$. Accordingly, the number of 3-faces equals the number of distinct 4 -tuples of vertexes, $\left(\begin{array}{c}k+1 \\ 4\end{array}\right)=(k+1) k(k-1)(k-2) / 4$ !.

In general, any $j$-tet of vertexes, $\mathrm{V}_{\mathrm{i}_{1}} \mathrm{~V}_{\mathrm{i}_{2}} \ldots \mathrm{V}_{\mathrm{i}_{\mathrm{j}}}, i_{1} \neq i_{2} \neq \ldots \neq i_{j}, 1 \leq j \leq k$, yields a $j$-hedron or $(j-1)$-face of $\Phi^{k+1}$. Accordingly, the number of $(j-1)$ faces equals the number of distinct $j$-tuples of vertexes, $\left(\begin{array}{c}k+1 \\ j\end{array}\right)=(k+1) k \ldots(k-$ $j+2) / j$ !

To this respect, a useful recursion formula follows from the identity:

$$
\left(\begin{array}{c}
k+1 \\
j
\end{array}\right)=\left(\begin{array}{c}
k \\
j
\end{array}\right)+\left(\begin{array}{c}
k \\
j-1
\end{array}\right) ; \quad 1 \leq j \leq k
$$

which translates into:

$$
F_{j}\left(\Phi^{k+1}\right)=F_{j}\left(\Phi^{k}\right)+F_{j-1}\left(\Phi^{k}\right) ; \quad 1 \leq j \leq k ;
$$

where, in general, $F_{\ell}\left(\Phi^{m+1}\right)$ is the number of $(\ell-1)$-faces of $\Phi^{m+1}, 1 \leq \ell \leq m$.

\section{C.3 A special case: $\Psi_{12}^{n}$ regular inclined $n$-hedrons}

With regard to an Euclidean $n$-space, $\Re^{n}$, and a Cartesian orthogonal reference frame, $\left(\mathrm{O} x_{1} x_{2} \ldots x_{n}\right)$, let a regular, inclined, $n$-hedron where vertexes are placed on the coordinate axes at a distance, $x_{i}=12,1 \leq i \leq 12$, from the origin, be considered and denoted as $\Psi_{12}^{n}$. Points with positive integer coordinates, or positive integer points, within $\Psi_{12}^{n}$ satisfy Eq. (1) as shown in the text. More specifically, the extension of Eq. (1) to real coordinates reads:

$$
p_{n}: \quad x_{1}+x_{2}+\ldots+x_{n}=12 ; \quad 1 \leq n \leq 12 ;
$$

which represents a $(n-1)$-plane where the intercepts on the coordinate axes coincide with the vertexes of $\Psi_{12}^{n}$.

The $n$-sector ( $n=2$, bisector; $n=3$, trisector; and so on) of the positive $2^{n}$-ant, by definition, includes both the origin and the point of unit coordinates, which implies $x_{i 0}=0, \bar{x}_{i 0}=1,1 \leq i \leq n$, and Eq. (866) reduces to:

$$
r_{n}: \quad x_{1}=x_{2}=\ldots=x_{n} ; \quad 1 \leq n \leq 12 ;
$$

which is the locus of points with identical coordinates. 
The angle between the $(n-1)$-plane, $p_{n}$, and the $n$-sector, $r_{n}$, keeping in mind $a_{i}=1, L_{i}=1,1 \leq i \leq n$, via Eq. (92) reads:

$$
\left|\sin \widehat{r_{n} p_{n}}\right|=\left|\frac{n}{\sqrt{n} \sqrt{n}}\right|=1 ;
$$

which implies $r_{n}$ is orthogonal to $p_{n}$ through the orthocentre of $\Psi_{12}^{n}$ that is, in fact, the sole point of $p_{n}$ with identical coordinates.

With regard to the generic $(j-1)$-face, $1 \leq j \leq n$, belonging to $\Psi_{12}^{n}$, the orthocentre, $\mathrm{H}_{\mathrm{j}}$, exhibits $j$ equal coordinates in that it is equally distant from $j$ vertexes of $\Psi_{12}^{n}$. Accordingly, nonzero coordinates equal $12 / j$ via Eq. (1). The result is:

$$
\begin{gathered}
\mathrm{H}_{\mathrm{j}}\left(i_{1}, i_{2}, \ldots, i_{j}\right) \equiv\left[\frac{12}{j} \sum_{k=1}^{j} \delta_{i_{k} 1}, \frac{12}{j} \sum_{k=1}^{j} \delta_{i_{k} 2}, \ldots, \frac{12}{j} \sum_{k=1}^{j} \delta_{i_{k} j}\right] ; \\
1 \leq i_{1}<i_{2}<\ldots<i_{j} \leq n ;
\end{gathered}
$$

where regular vertexes, regular sides, regular triangles, regular tetrahedrons, and so on, are conceived as 0-faces, 1-faces, 2-faces, 3-faces, and so on, respectively. In particular, the orthocentre of $\Psi_{12}^{n}$, conceived as related to a single $(n-1)$-face, reads:

$$
\mathrm{H}_{\mathrm{n}}(1,2, \ldots, n) \equiv\left(\frac{12}{n}, \frac{12}{n}, \ldots, \frac{12}{n}\right) ;
$$

where $\mathrm{H}_{\mathrm{n}}$ is a positive integer point only if the ratio, $12 / n$, is integer, which implies $n=1,2,3,4,6,12$.

The number of $(j-1)$-faces, $F_{j}, 1 \leq j \leq n$, belonging to $\Psi_{12}^{n}, 1 \leq n \leq 13$, can be inferred from the general results mentioned above, where $k=n-1$, as listed in Table 7. More specifically, defining $(-1)$-faces and $(n-1)$-faces as $n$-hedron metacentres and $n$-hedrons, respectively, related values equal unity and Table 7 reproduces Pascal's triangle e.g., [15] §30.

The metacentre of $\Psi_{12}^{n}$ is defined as the centre of a $n$-sphere, on the surface of which vertexes of $\Psi_{12}^{n}$ are lying i.e. the origin of coordinates. In addition, the metacentre can be thought of as the orthocentre of a $(n+1)$-hedron where $\Psi_{12}^{n}$ is a $(n-1)$-face and the additional vertex lies on the $n$-sector of the negative $2^{n}$-ant, at a distance, $R_{n}=12$, from the origin.

In the special case, $n=0$, related 0 -hedron lies outside $\Re^{0}$ and no vertex can be defined, while the metacentre coincides with $\Re^{0}$, yielding $1(-1)$-face.

Let a $(j-1)$-face and a $(n-j-1)$-face of $\Psi_{12}^{n}$ be assigned. The coordinates of generic points lying within, $P_{j}, P_{n-j}$, respectively, read:

$$
\mathrm{P}_{\mathrm{j}} \equiv\left(\ell_{1} \sum_{k=1}^{j} \delta_{i_{k} 1}, \ell_{2} \sum_{k=1}^{j} \delta_{i_{k} 2}, \ldots, \ell_{n} \sum_{k=1}^{j} \delta_{i_{k} j}\right) ;
$$


Table 7: Number of $(j-1)$-faces, $F_{j}, 0 \leq j \leq n$, belonging to $\Psi_{12}^{n}, 0 \leq n \leq 13$. In particular, $n=F_{1}$. The addition of $(-1)$-faces, $F_{0}$, related to $n$-hedron metacentres, and $(n-1)$-faces, $F_{n}$, related to $n$-hedrons, makes the horizontal and oblique unit line, respectively, yielding Pascal's triangle. For $j<10, F_{j}$ is denoted as $F_{0 j}$ to save aesthetics. See text for further details.

\begin{tabular}{|l|l|l|l|l|l|r|r|r|r|r|r|r|r|r|}
\hline \hline$F_{00}$ & 1 & 1 & 1 & 1 & 1 & 1 & 1 & 1 & 1 & 1 & 1 & 1 & 1 & 1 \\
$F_{01}$ & & 1 & 2 & 3 & 4 & 5 & 6 & 7 & 8 & 9 & 10 & 11 & 12 & 13 \\
$F_{02}$ & & & 1 & 3 & 6 & 10 & 15 & 21 & 28 & 36 & 45 & 55 & 66 & 78 \\
$F_{03}$ & & & 1 & 4 & 10 & 20 & 35 & 56 & 84 & 120 & 165 & 220 & 286 \\
$F_{04}$ & & & & 1 & 5 & 15 & 35 & 70 & 126 & 210 & 330 & 495 & 715 \\
$F_{05}$ & & & & & 1 & 6 & 21 & 56 & 126 & 252 & 462 & 792 & 1287 \\
$F_{06}$ & & & & & & 1 & 7 & 28 & 84 & 210 & 462 & 924 & 1716 \\
$F_{07}$ & & & & & & & 1 & 8 & 36 & 120 & 330 & 792 & 1716 \\
$F_{08}$ & & & & & & & & 9 & 45 & 165 & 495 & 1287 \\
$F_{09}$ & & & & & & & & 1 & 10 & 55 & 220 & 715 \\
$F_{10}$ & & & & & & & & & 1 & 11 & 66 & 286 \\
$F_{11}$ & & & & & & & & & & & 1 & 12 & 78 \\
$F_{12}$ & & & & & & & & & & & & 1 & 13 \\
$F_{13}$ & & & & & & & & & & & & & 1 \\
\hline
\end{tabular}




$$
\begin{gathered}
1 \leq i_{1}<i_{2}<\ldots<i_{j} \leq n ; \\
\mathrm{P}_{\mathrm{n}-\mathrm{j}} \equiv\left(\begin{array}{c}
\left.\ell_{1}^{\prime} \sum_{m=1}^{n-j} \delta_{i_{m}^{\prime} 1}, \ell_{2}^{\prime} \sum_{m=1}^{n-j} \delta_{i_{m}^{\prime} 2}, \ldots, \ell_{n}^{\prime} \sum_{m=1}^{n-j} \delta_{i_{m}^{\prime} j}\right) ; \\
1 \leq i_{1}^{\prime}<i_{2}^{\prime}<\ldots<i_{n-j}^{\prime} \leq n
\end{array}\right.
\end{gathered}
$$

and the hyperfaces under discussion are defined as opposite if, in addition, $i_{k} \neq i_{m}^{\prime}, 1 \leq k \leq j, 1 \leq m \leq n-j$.

The special case, $P_{j} \equiv H_{j}, P_{n-j} \equiv H_{n-j}$, via Eq. (98) reads:

$$
\begin{aligned}
& \mathrm{H}_{\mathrm{j}} \equiv\left(\frac{12}{j} \sum_{k=1}^{j} \delta_{i_{k} 1}, \frac{12}{j} \sum_{k=1}^{j} \delta_{i_{k} 2}, \ldots, \frac{12}{j} \sum_{k=1}^{j} \delta_{i_{k} n}\right) ; \\
& 1 \leq i_{1}<i_{2}<\ldots<i_{j} \leq n ; \\
& \mathrm{H}_{\mathrm{n}-\mathrm{j}} \equiv\left(\frac{12}{n-j} \sum_{m=1}^{n-j} \delta_{i_{m}^{\prime} 1}, \frac{12}{n-j} \sum_{m=1}^{n-j} \delta_{i_{m}^{\prime} 2}, \ldots, \frac{12}{n-j} \sum_{m=1}^{n-j} \delta_{i_{m}^{\prime} n}\right) ; \\
& 1 \leq i_{1}^{\prime}<i_{2}^{\prime}<\ldots<i_{n-j}^{\prime} \leq n
\end{aligned}
$$

where $i_{k} \neq i_{m}^{\prime} ; 1 \leq k \leq j ; 1 \leq m \leq n-j$; and the orthocentre of $\psi_{12}^{n}, \mathrm{H}_{\mathrm{n}}$, is necessarily aligned with $\mathrm{H}_{\mathrm{j}}$ and $\mathrm{H}_{\mathrm{n}-\mathrm{j}}$.

More specifically, the particularization of Eq. (86) to $x_{i 0}=(12 / j)\left(\delta_{i_{1} i}+\right.$ $\left.\delta_{i_{2} i}+\ldots+\delta_{i_{j} i}\right), \bar{x}_{i 0}=[12 /(n-j)]\left(\delta_{i_{1}^{\prime} i}+\delta_{i_{2}^{\prime} i}+\ldots+\delta_{i_{n-j}^{\prime}} i\right), 1 \leq i \leq n$, yields:

$$
\begin{gathered}
\frac{x_{1}-\frac{12}{j} \sum_{k=1}^{j} \delta_{i_{k} 1}}{\frac{12}{n-j} \sum_{m=1}^{n-j} \delta_{i_{m}^{\prime} 1}-\frac{12}{j} \sum_{k=1}^{j} \delta_{i_{k} 1}}=\frac{\frac{12}{n-j} \sum_{m=1}^{n-j} \delta_{i_{m}^{\prime} 1}-\frac{12}{j} \sum_{k=1}^{j} \delta_{i_{k} 1} \delta_{i_{k} 1}}{x_{n=1}-\frac{12}{j} \sum_{k=1}^{j} \delta_{i_{k} n}}=\ldots \\
=\frac{\frac{12}{n-j} \sum_{m=1}^{n-j} \delta_{i_{m}^{\prime} n}-\frac{12}{j} \sum_{k=1}^{j} \delta_{i_{k} n}}{} ;
\end{gathered}
$$

where, in the case under discussion of opposite hyperfaces, each sum equals zero or unity and attains different values on different points. Accordingly, the generic term appearing in Eq. (102) reads either $-j x_{i} / 12+1$, for a total of $j$ occurrences, or $(n-j) x_{i} / 12$, for a total of $(n-j)$ occurrences, $1 \leq i \leq n$.

In the special case of the orthocentre of $\Psi_{12}^{n}, x_{i}=12 / n, 1 \leq i \leq n$, and the above mentioned alternatives reduce to either $-(j / 12)(12 / n)+1=(n-j) / n$ or $[(n-j) / 12](12 / n)=(n-j) n$, which implies Eq. (102) is satisfied and, 
in turn, the orthocentre of $\Psi_{12}^{n}$ is aligned with the orthocentre of opposite $(j-1)$ and $(n-j-1)$-faces.

Let $\mathrm{P}_{\mathrm{n}} \equiv\left(\ell_{1}, \ell_{2}, \ldots, \ell_{n}\right)$ be a generic integer point belonging to $\Psi_{12}^{n}$. The square distance, $\left(\overline{\mathrm{P}_{\mathrm{n}} \mathrm{H}_{\mathrm{n}}}\right)^{2}=R_{n}^{2}$, from the orthocentre of $\Psi_{12}^{n}$, via Eqs. (84) and (99) reads:

$$
R_{n}^{2}=\sum_{i=1}^{n}\left(\ell_{i}-\ell_{0}\right)^{2} \quad ; \quad \ell_{0}=\frac{12}{n} ;
$$

where $R_{n}$ is the radius of a $(n-1)$-circle centered on $\mathrm{H}_{\mathrm{n}}$.

The straight line joining $P_{n}$ and $H_{n}$, via Eq. (86) is expressed as:

$$
r_{a}: \quad \frac{x_{1}-\ell_{0}}{\ell_{1}-\ell_{0}}=\frac{x_{2}-\ell_{0}}{\ell_{2}-\ell_{0}}=\ldots=\frac{x_{n}-\ell_{0}}{\ell_{n}-\ell_{0}} ;
$$

where $L_{k}=\ell_{k}-\ell_{0}, 1 \leq k \leq n$.

The counterpart of Eq. (104), related to a selected permutation of the coordinates of $P_{n}$, is:

$$
r_{b}: \quad \frac{x_{1}-\ell_{0}}{\ell_{i_{1}}-\ell_{0}}=\frac{x_{2}-\ell_{0}}{\ell_{i_{2}}-\ell_{0}}=\ldots=\frac{x_{n}-\ell_{0}}{\ell_{i_{n}}-\ell_{0}}
$$

where $L_{i_{k}}=\ell_{i_{k}}-\ell_{0}, 1 \leq k \leq n$.

The angle, $\widehat{r_{a} r_{b}}$, via Eq. (90) reads:

$$
\left|\cos \widehat{r_{a} r_{b}}\right|=\left|\frac{\sum_{k=1}^{n}\left(\ell_{k}-\ell_{0}\right)\left(\ell_{i_{k}}-\ell_{0}\right)}{\left[\sum_{i=1}^{k}\left(\ell_{k}-\ell_{0}\right)^{2}\right]^{1 / 2}\left[\sum_{i=1}^{k}\left(\ell_{i_{k}}-\ell_{0}\right)^{2}\right]^{1 / 2}}\right| ;
$$

where, in the case under discussion, the sums of squares exhibit same addends in different order. Accordingly, Eq. (106) reduces to:

$$
\left|\cos \widehat{r_{a} r_{b}}\right|=\left|\frac{\sum_{k=1}^{n}\left(\ell_{k}-\ell_{0}\right)\left(\ell_{i_{k}}-\ell_{0}\right)}{\sum_{i=1}^{k}\left(\ell_{k}-\ell_{0}\right)^{2}}\right| ;
$$

where the permutation of the coordinates acts only on the sum of products.

The special case of circular permutation, $i_{k}=k+1$, reads:

$$
\left|\cos \widehat{r_{a} r_{b}}\right|=\left|\frac{\sum_{k=1}^{n}\left(\ell_{k}-\ell_{0}\right)\left(\ell_{k+1}-\ell_{0}\right)}{\sum_{i=1}^{k}\left(\ell_{k}-\ell_{0}\right)^{2}}\right| ;
$$


where $\ell_{n+1}=\ell_{1}$. It is apparent adjacent circular permutations of coordinates e.g., $\left(\ell_{1}, \ell_{2}, \ldots, \ell_{n}\right),\left(\ell_{2}, \ell_{3}, \ldots, \ell_{1}\right) ;\left(\ell_{2}, \ell_{3}, \ldots, \ell_{1}\right),\left(\ell_{3}, \ell_{4}, \ldots, \ell_{2}\right)$; yield the same angle in that the sum of products in Eq. (108) exhibits same addends in different order. On the other hand, non adjacent circular permutations of coordinates yield different angles with respect to the sum of adjacent angles, as related straight lines are not complanar. In fact, positive integer points whose coordinates belong to a same $T_{n}$ set class are similarly placed within different $\Psi_{12, i}^{n}, 1 \leq i \leq n$, i.e. congruent $(n-1)$-hedrons, where the $i$ th vertex coincides with the orthocentre of $\Psi_{12}^{n}$ and the remaining ones coincide with vertexes (different from the $i$ th) of $\Psi_{12}^{n}$.

The special case of reflection, $i_{k}=n-k+1$, reads:

$$
\left|\cos \widehat{r_{a} r_{b}}\right|=\left|\frac{\sum_{k=1}^{n}\left(\ell_{k}-\ell_{0}\right)\left(\ell_{n-k+1}-\ell_{0}\right)}{\sum_{i=1}^{k}\left(\ell_{k}-\ell_{0}\right)^{2}}\right| ;
$$

where adjacent circular permutations yield the same angle as the sum of products in Eq. (109) exhibits same addends in different order. In fact, positive integer points with coordinates belonging to a same $T_{n} / T_{n} I$ set class are similarly and symmetrically placed within different $\Psi_{12, i}^{n}, 1 \leq i \leq n$.

The mean point, $M_{n}$, of the segment joining pairwise points, $P_{n} \equiv\left(\ell_{1}, \ell_{2}, \ldots, \ell_{n}\right)$, $\mathrm{Q}_{\mathrm{n}} \equiv\left(\ell_{n}, \ell_{n-1}, \ldots, \ell_{1}\right)$, via Eq. (85) reads:

$$
\mathrm{M}_{\mathrm{n}} \equiv\left(\frac{\ell_{1}+\ell_{n}}{2}, \frac{\ell_{2}+\ell_{n-1}}{2}, \ldots, \frac{\ell_{n}+\ell_{1}}{2},\right)
$$

accordingly, the coordinates of $\mathrm{M}_{\mathrm{n}}$ are palindrome as either $\left(a_{1}, \ldots, a_{n / 2}, a_{n / 2}, \ldots, a_{1}\right)$ or $\left(a_{1}, \ldots, a_{(n-1) / 2}, a_{(n+1) / 2}, a_{(n-1) / 2}, \ldots, a_{1}\right)$ according if $n$ is even or odd, respectively.

To get further insight, let $\Psi_{12}^{n}$ be conceived as a series of Chinese-box $n$-hedronical shells, $\psi_{k, 12}^{n}$, where the external shell, $\psi_{0,12}^{n}$, has not to be considered in that related points exhibit one null coordinate at least. The last shell of the series relates to the value of $k$ for which the condition, $k \leq 12 / n$, still holds. The special case, $k=12 / n$, implies $\psi_{12 / n, 12}^{n}$ coincides with the orthocentre of $\Psi_{12}^{n}$, provided $n$ is a divisor of 12 . For instance, vertexes of $\psi_{k, 12}^{n} \operatorname{read} \mathrm{V}_{\mathrm{i}}^{\prime} \equiv\left[k+\delta_{i 1}(12-k n), k+\delta_{i 2}(12-k n), \ldots, k+\delta_{i n}(12-k n)\right]$, and mean points between vertexes read $\mathrm{M}_{\mathrm{ij}}^{\prime} \equiv\left[k+\left(\delta_{i 1}+\delta_{j 1}\right)(12-k n) / 2, k+\left(\delta_{i 2}+\right.\right.$ $\left.\left.\delta_{j 2}\right)(12-k n) / 2, \ldots, k+\left(\delta_{i n}+\delta_{j n}\right)(12-k n) / 2\right]$.

For even $n$, the straight line joining mean points on opposite side of $\psi_{k, 12}^{n}$, 
restricting to palindrome coordinates, via Eq. (86) is expressed as:

$$
\begin{aligned}
& \frac{x_{n / 2-1}-k}{[k+(12-k n) / 2]-k}=\frac{x_{n / 2}-[k+(12-k n) / 2]}{k-[k+(12-k n) / 2]} \\
& =\frac{x_{n / 2+1}-[k+(12-k n) / 2]}{k-[k+(12-k n) / 2]}=\frac{x_{n / 2+2}-k}{[k+(12-k n) / 2]-k} ;
\end{aligned}
$$

which, after some algebra, reduces to:

$$
x_{n / 2-1}=-x_{n / 2}+2 k+6-\frac{k n}{2}=-x_{n / 2+1}+2 k+6-\frac{k n}{2}=x_{n / 2+2} ;
$$

while the remaining coordinates are fixed as:

$$
x_{1}=\ldots=x_{n / 2-2}=x_{n / 2+3}=\ldots=x_{n}=k ;
$$

in connection with the selected $\psi_{k, 12}^{n}$.

In conclusion, positive integer points exhibiting palindrome coordinates, for even $n$ lie on the straight line, expressed by Eqs. (112)-(113). For instance, let $n=6, k=1$, be considered. Accordingly, Eqs. (112)-(113) reduce to:

$$
x_{2}=-x_{3}+5=-x_{4}+5=x_{5} ; \quad x_{1}=x_{6}=1 ;
$$

where $(1,4,1,1,4,1),(1,1,4,4,1,1)$, are coordinates of mean points on opposite sides of related 4 -face, and $(1,3,2,2,3,1),(1,2,3,3,2,1)$, are palindrome coordinates of positive integer points on the straight line joining the above mentioned mean points, expressed by Eq. (114).

For odd $n$, the straight line joining a vertex with the mean point of the opposite sides of a 3 -face of $\psi_{k, 12}^{n}$, restricting to palindrome coordinates, via Eq. (86) is expressed as:

$$
\begin{aligned}
\frac{x_{(n-1) / 2}-k}{[k+(12-k n) / 2]-k} & =\frac{x_{(n+1) / 2}-[k+(12-k n)]}{k-[k+(12-k n) / 2]} \\
& =\frac{x_{(n+3) / 2}-k}{[k+(12-k n) / 2]-k}
\end{aligned}
$$

which, after some algebra, reduces to:

$$
2 x_{(n-1) / 2}=-x_{(n+1) / 2}+12-k n+3 k=2 x_{(n+3) / 2} ;
$$

while the remaining coordinates are fixed as:

$$
x_{1}=\ldots=x_{(n-3) / 2}=x_{(n+5) / 2}=\ldots=x_{n}=k ;
$$


in connection with the selected $\psi_{k, 12}^{n}$.

In conclusion, positive integer points exhibiting palindrome coordinates, for odd $n$ lie on the straight line, expressed by Eqs. (116)-(117). For instance, let $n=5, k=1$, be considered. Accordingly, Eqs. (116)-(117) reduce to:

$$
2 x_{2}=-x_{3}+10=2 x_{4} \quad ; \quad x_{1}=x_{5}=1 ;
$$

where $(1,1,8,1,1),(1,9 / 2,1,9 / 2,1)$, are coordinates of the vertex and mean point on opposite side of related 3 -face, respectively, and $(1,2,6,2,1),(1,3,4,3,1)$, $(1,4,2,4,1)$, are palindrome coordinates of positive integer points on the straight line joining the above mentioned vertex and mean point, expressed by Eq. (118). 


\begin{tabular}{|c|c|c|c|c|c|c|c|c|c|}
\hline 01,01 & 01,02 & 01,03 & 01,04 & 01,05 & 01,06 & 01,07 & 01,08 & 01,09 & 01,10 \\
\hline 02,01 & 02,02 & 02,03 & 02,04 & 02,05 & 02,06 & 02,07 & 02,08 & 02,09 & 02,10 \\
\hline 03,01 & 03,02 & 03,03 & 03,04 & 03,05 & 03,06 & 03,07 & 03,08 & 03,09 & 03,10 \\
\hline 04,01 & 04,02 & 04,03 & 04,04 & 04,05 & 04,06 & 04 & 04,08 & 04,09 & 04,10 \\
\hline 05,01 & 05,02 & 05,03 & 05,04 & 05,05 & & 05,07 & 05,08 & 05,09 & 05,10 \\
\hline 06,01 & 06,02 & 06,03 & 06,04 & 06 & 6 & 06,07 & 06,08 & 06,09 & 06,10 \\
\hline 07,01 & 07,02 & 07,03 & 07,04 & 07,05 & 07,06 & 07,07 & 07,08 & 07,09 & 07,10 \\
\hline 08,01 & 08,02 & 08,03 & 08,04 & 08,05 & 08,06 & 08,07 & 08,08 & 08,09 & 08,10 \\
\hline 09,01 & 09,0 & 09,03 & 09,04 & 09,05 & 09,06 & 09,07 & 09,08 & 09,09 & 09,10 \\
\hline 10,01 & 10,02 & 10,03 & 10,04 & 10,05 & 10,06 & 10,07 & 10,08 & 10,09 & 10,10 \\
\hline 11,01 & 11,02 & 11,03 & 11,04 & 11,05 & 11,06 & 11,07 & 11,08 & 11,09 & 11,10 \\
\hline
\end{tabular}

Figure 1: The 1: 1 correspondence between projected points, $P_{n-1} \leftrightarrow Q_{n-1}$, in the special case of an Euclidean $n$-space, $n=3, \mathrm{P}_{2}\left(\ell_{1}, \ell_{2}\right) \leftrightarrow \mathrm{Q}_{2}\left(12-\ell_{1}, \ell_{2}\right)$, yielding a total number of distinct $n$-chords, $N_{\mathrm{C}}(3)=55$. The coordinates of projected points, $P_{2}$, satisfying Eq. (2) i.e. $\ell_{3}=12-\ell_{1}-\ell_{2}$, are placed above the straight line with positive slope. The coordinates of projected points, $\mathrm{Q}_{2} \leftrightarrow \mathrm{P}_{2}$, are placed below the straight line with negative slope. The coordinates of projected points, $\mathrm{P}_{2} \leftrightarrow \mathrm{Q}_{2}, \mathrm{P}_{2}^{\prime} \leftrightarrow \mathrm{Q}_{2}^{\prime}, \mathrm{P}_{2} \equiv \mathrm{Q}_{2}^{\prime}, \mathrm{P}_{2}^{\prime} \equiv \mathrm{Q}_{2}$, are placed within the left angle bisected by a horizontal straight line. The coordinates of points for which the correspondence does not hold and Eq. (2) is violated i.e. $\ell_{3}>12-\ell_{1}-\ell_{2}$, are placed within the right angle bisected by a horizontal line. In representing coordinates, brackets have been omitted to save space. The correspondence, $\mathrm{P}_{2} \leftrightarrow \mathrm{Q}_{2}$, acts along columns. Initial zeroes have been preferred to blank spaces to save aesthetics. 


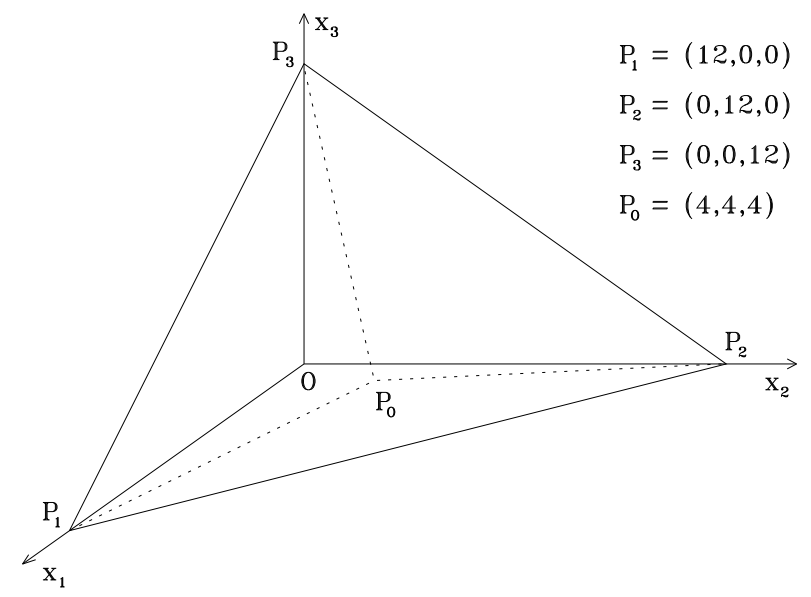

Figure 2: The special case, $n=3$, where the regular inclined $n$-hedron reduces to a regular triangle and congruent $n$-hedrons to isosceles triangles with adjacent equal sides. The common vertex of isosceles triangles coincides with the orthocentre of the regular triangle. 


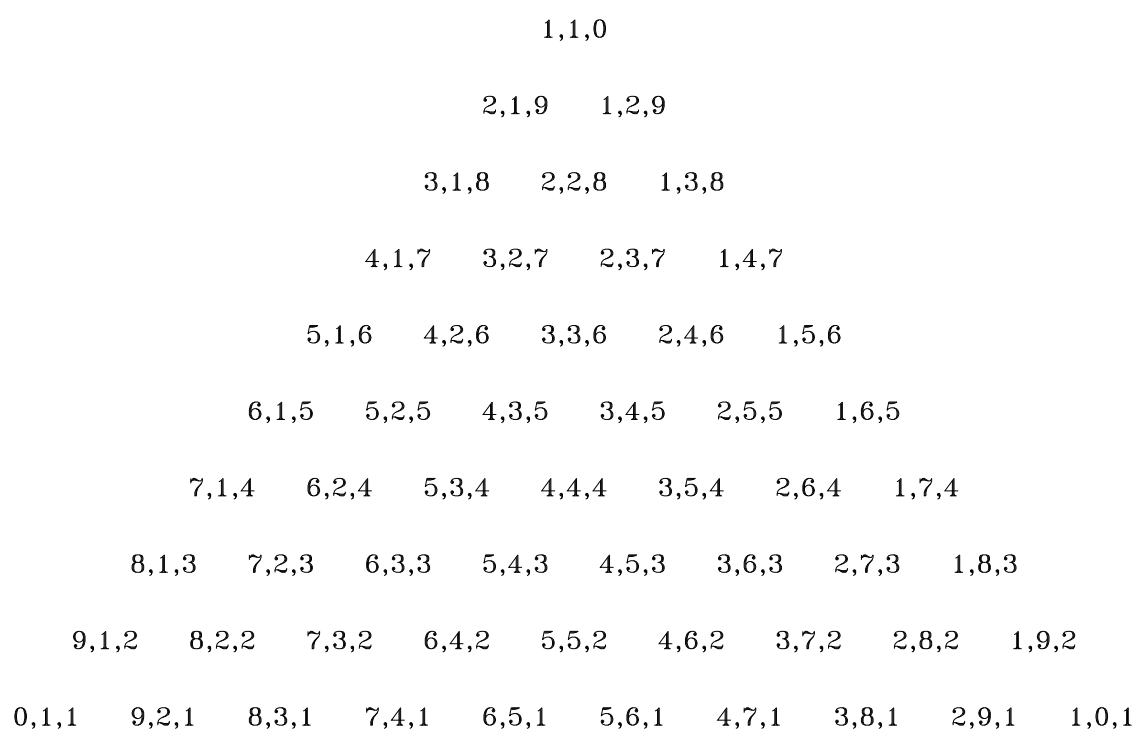

Figure 3: Representation of distinct $n$-chords of cardinality, $n$, as positive integer coordinates of points in an Euclidean $n$-space, lying within a regular inclined $n$-hedron via Eq. (1), in the special case, $n=3$. The total number of distinct 3 -chords is $N_{\mathrm{C}}(3)=55$. In representing coordinates, brackets have been omitted to save space and ten has been replaced by zero to save aesthetics. 